\title{
Constrained Source-Coding With Side Information
}

\author{
Amos Lapidoth, Fellow, IEEE, Andreas Malär, and Michèle Wigger, Member, IEEE
}

\begin{abstract}
The source-coding problem with side information at the decoder is studied subject to a constraint that the encoderto whom the side information is unavailable-be able to compute the decoder's reconstruction sequence to within some distortion. For discrete memoryless sources and finite single-letter distortion measures, an expression is given for the minimal description rate as a function of the joint law of the source and side information and of the allowed distortions at the encoder and at the decoder. The minimal description rate is also computed for a memoryless Gaussian source with squared-error distortion measures. A solution is also provided to a more general problem where there are more than two distortion constraints and each distortion measure may be a function of three arguments: the source symbol, the encoder's reconstruction symbol, and the decoder's reconstruction symbol.
\end{abstract}

Index Terms-Source coding, Wyner-Ziv coding, side information, rate-distortions function, constrained reconstructions.

\section{INTRODUCTION}

$\mathbf{L}$ IKE Wyner and Ziv [1], we study a setting where a sequence generated by a source is to be described succinctly to a reconstructor ("decoder") with access to some side information. Wyner and Ziv showed that, although the side information is not available at the describing terminal ("encoder"), it can be beneficial in improving the trade-off between the rate of description and the reconstruction distortion. They fully characterized this trade-off for memoryless sources with single-letter distortion measures. Unlike the case without side information - since the side information is used in the reconstruction process, and since the side information is not available at the describing terminal-the describing terminal cannot tell how the source sequence it observes will be reconstructed. In some settings, this is unacceptable. Steinberg [2] therefore studied the common-reconstruction problem where an additional restriction is imposed that the reconstruction sequence be computable with probability nearly one at the describing terminal. This greatly limits the extent by

Manuscript received January 22, 2013; revised December 9, 2013; accepted February 10, 2014. Date of publication April 1, 2014; date of current version May 15, 2014. A. Malär was supported by an IDEA League Student Grant. M. Wigger was supported by the Emergences Programme, City of Paris. This paper was presented at the 2011 Information Theory and Applications Workshop and the 2011 IEEE International Symposium on Information Theory.

A. Lapidoth is with the Department of Information Technology and Electrical Engineering, ETH Zurich, Zurich 8092, Switzerland (e-mail: lapidoth@isi.ee.ethz.ch).

A. Malär was with the Department of Information Technology and Electrical Engineering, ETH Zurich, Zurich 8092, Switzerland. He is now with Malcom AG, Zurich 8037, Switzerland (e-mail: andreas@malcom.ch).

M. Wigger is with the Communications and Electronics Department, Telecom ParisTech, Paris 75013, France (e-mail: michele.wigger@telecomparistech.fr).

Communicated by Y. Liang, Associate Editor for Shannon Theory.

Digital Object Identifier 10.1109/TIT.2014.2314634

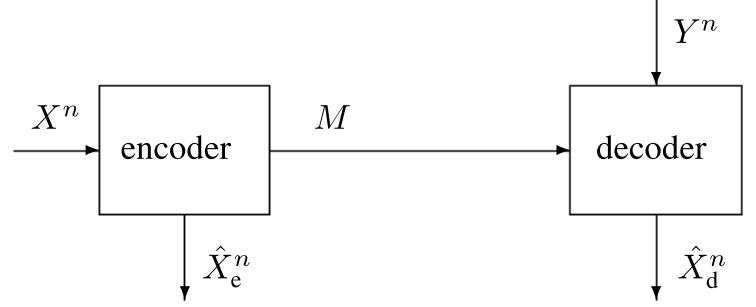

$\frac{1}{n} \sum_{i=1}^{n} \mathrm{E}\left[d_{\mathrm{e}}\left(\hat{X}_{\mathrm{d}, i}, \hat{X}_{\mathrm{e}, i}\right)\right] \leq D_{\mathrm{e}} ; \quad \frac{1}{n} \sum_{i=1}^{n} \mathrm{E}\left[d_{\mathrm{d}}\left(X_{i}, \hat{X}_{\mathrm{d}, i}\right)\right] \leq D_{\mathrm{d}}$

Fig. 1. Constrained Wyner-Ziv coding.

which the reconstruction can depend on the side information. More generally, there is a tension between the degree by which the reconstructing terminal utilizes the side information and the precision with which the describing terminal can compute the reconstruction sequence. It is this tension that we study in this paper.

To quantify this tension, we require that the describing terminal generate an estimate of the sequence that will be produced at the reconstructing terminal (Figure 1). We then study the distortions that can be simultaneously achieved at the describing terminal ("the encoder distortion") and at the reconstructing terminal ("the decoder distortion") as a function of the description rate. If the encoder's distortion measure is the Hamming distance and if the allowed distortion is zero, then our problem reduces in essence to Steinberg's commonreconstruction problem. ${ }^{1}$ And if the allowed encoder distortion is infinite, our problem reduces to that of Wyner and Ziv. We can thus view our problem as a generalization of the WynerZiv problem and Steinberg's common reconstruction problem.

For discrete memoryless sources and finite single-letter distortion measures, we provide a single-letter characterization of the trade-off between the description rate and the distortions at the encoder and decoder sides. We also calculate this trade-off for a memoryless Gaussian source and squared-error distortion measures. Finally, in Section IV, we generalize the results to account for more than two constraints and to allow each distortion measure to depend on three arguments: the source symbol, the encoder's reconstruction symbol, and the decoder's reconstruction symbol.

Steinberg's work was also extended in other ways. Kittichokechai, Oechtering, and Skoglund [3] determined the rate-distortion function under a common-reconstruction

\footnotetext{
${ }^{1}$ Steinberg used a vanishingly small block-error criterion whereas we use a vanishingly small average-per-symbol error criterion. See Remark 3 in Section II-B ahead.
} 
constraint for a modified Wyner-Ziv setup where the encoder can influence the decoder's side information via an action-generator. Ahmadi, Tandon, Simeone, and Poor [6] presented the rates-distortions function under a common-reconstruction constraint for a cascade source-coding problem when the side informations are physically degraded. Timo, Grant, and Kramer [4], [5], and Ahmadi, Tandon, Simeone, and Poor [6] derived the rate-distortions function under a common-reconstruction constraint for two special cases of the Heegard-Berger/Kaspi problem (the Wyner-Ziv problem with two decoders): [6] for physically degraded side informations, and [4], [5] for complementary side informations. Timo, Grant, and Kramer [5] extended their work also to a joint source-channel coding setup. Already in [2], Steinberg studied the implications of the commonreconstruction constraint on joint source-channel coding for the degraded broadcast channel and on the simultaneous transmission of data and state. Vellambi and Timo [7] finally studied the Heegard-Berger problem under a slightly modified common reconstruction constraint, where the two receivers are required to reconstruct each others reconstructions of the source. For this setup they derived the rate-distortions function when a) the side-information is physically degraded; b) the side information is stochastically degraded and a certain full-support condition holds; or c) also the encoder is required to reconstruct both receivers' reconstructions.

Our results in Section II ahead, have recently been extended by Rezagah and Erkip [8]. They studied the setup where the two terminals (here termed encoder and decoder) wish to reconstruct functions of the two sources (here termed source and side information) and where to achieve this goal they can alternatingly exchange messages during a given number of $t \geq 1$ rounds. (Our setup corresponds to $t=1$ rounds.) They gave a single-letter characterization of the rates-distortions regions for discrete-memoryless sources with average-persymbol distortion measures and for Gaussian sources with squared-error distortion measures.

The paper is organized as follows. In the rest of this section we introduce our notation. In Section II we treat discrete sources and general distortions, and in Section III Gaussian sources with quadratic distortions. In Section IV we revisit discrete sources but this time with more and more general distortion constraints.

\section{A. Notation}

Random variables are denoted by upper-case letters and their realizations by lower-case letters. Vectors are denoted by bold-face letters: random vectors by upper-case boldface letters, and deterministic vectors by lower-case boldface letters. The transpose of a vector $\mathbf{a}$ is denoted by $\mathbf{a}^{\top}$; its Euclidean norm by $\|\mathbf{a}\|$; and the Euclidean inner product between the vectors $\mathbf{a}$ and $\mathbf{b}$ by $\langle\mathbf{a}, \mathbf{b}\rangle$.

Sets and events are denoted by calligraphic letters, e.g., $\mathcal{A}$. An $n$-tuple $\left(A_{1}, \ldots, A_{n}\right)$ is denoted $A^{n}$, and the $n$-fold Cartesian product of the set $\mathcal{A}$ is denoted $\mathcal{A}^{n}$. The convex hull of a set $\mathcal{A}$ is denoted by $\operatorname{conv}(\mathcal{A})$, and its cardinality by $|\mathcal{A}|$. The set of real numbers is denoted $\mathbb{R}$ and its $d$-fold Cartesian product $\mathbb{R}^{d}$. The nonnegative reals are denoted $\mathbb{R}_{+}$, and the positive reals $\mathbb{R}_{++}$. For the respective $d$-fold Cartesian products we write $\mathbb{R}_{+}^{d}$ and $\mathbb{R}_{++}^{d}$.

To indicate that two random variables $A$ and $C$ are conditionally independent given a third random variable $B$ we write

$$
A \multimap-B \multimap C \text {. }
$$

The abbreviation IID stands for independently and identically distributed and w.p. 1 stands for with probability 1. Further, $E[\cdot]$ denotes the expectation operator.

We use $\mathbb{I}\{\cdot\}$ to denote the indicator function: $\mathbb{I}\{$ statement $\}$ is equal to one if the statement is true and is equal to zero if it is false. Throughout the paper $\log (\cdot)$ denotes base-2 logarithm, and $\log ^{+}(\xi)=\max \{\log \xi, 0\}$.

\section{Discrete Memoryless Sources AND GENERAL DisTORTIONS}

\section{A. Problem Statement}

Our setting is illustrated in Figure 1 and is specified by a tuple

$$
\left(\mathcal{X}, \mathcal{Y}, \hat{\mathcal{X}}, P_{X Y}, d_{\mathrm{d}}, d_{\mathrm{e}}, D_{\mathrm{d}}, D_{\mathrm{e}}\right)
$$

where $\mathcal{X}, \mathcal{Y}, \hat{\mathcal{X}}$ are finite sets; $P_{X Y}$ is a probability distribution on $\mathcal{X} \times \mathcal{Y} ; d_{\mathrm{d}}(\cdot, \cdot)$ and $d_{\mathrm{e}}(\cdot, \cdot)$ are nonnegative functions

$$
\begin{aligned}
& d_{\mathrm{d}}: \mathcal{X} \times \hat{\mathcal{X}} \rightarrow \mathbb{R}^{+} \\
& d_{\mathrm{e}}: \hat{\mathcal{X}} \times \hat{\mathcal{X}} \rightarrow \mathbb{R}^{+}
\end{aligned}
$$

and $D_{\mathrm{d}}$ and $D_{\mathrm{e}}$ are nonnegative real numbers.

The sets $\mathcal{X}, \mathcal{Y}$, and $\hat{\mathcal{X}}$ are the source, side information, and reconstruction alphabets. A source sequence $X^{n} \in \mathcal{X}^{n}$ is observed at the encoder (but not at the decoder) and a sideinformation sequence $Y^{n} \in \mathcal{Y}^{n}$ at the decoder (but not at the encoder). The sequence of pairs $\left\{\left(X_{i}, Y_{i}\right)\right\}_{i=1}^{n}$ is assumed to be drawn IID according to the joint law $P_{X Y}$.

The encoder describes the source sequence $X^{n}$ to the decoder by an index

$$
M=f^{(n)}\left(X^{n}\right)
$$

where

$$
f^{(n)}: \mathcal{X}^{n} \rightarrow \mathcal{M}
$$

is the encoding function and

$$
\mathcal{M} \triangleq\{1, \ldots, \mathrm{M}\}
$$

denotes the message set for some positive integer $\mathrm{M}$. Based on the index $M$ and its side information $Y^{n}$, the decoder forms a reconstruction sequence

$$
\hat{X}_{\mathrm{d}}^{n}=\phi^{(n)}\left(M, Y^{n}\right)
$$

where

$$
\phi^{(n)}: \mathcal{M} \times \mathcal{Y}^{n} \rightarrow \hat{\mathcal{X}}^{n}
$$

is the decoder's reconstruction function. The encoder's estimate of the decoder's reconstruction sequence is

$$
\hat{X}_{e}^{n}=\psi^{(n)}\left(X^{n}\right)
$$


for some

$$
\psi^{(n)}: \mathcal{X}^{n} \rightarrow \hat{\mathcal{X}}^{n}
$$

The goal is that the decoder's reconstruction $\hat{X}_{\mathrm{d}}^{n}$ be within distortion $D_{\mathrm{d}}$ of the source sequence $X^{n}$ and that the encoder's estimate $\hat{X}_{\mathrm{e}}^{n}$ be within distortion $D_{\mathrm{e}}$ from the decoder's reconstruction $\hat{X}_{\mathrm{d}}^{n}$. The distortions are measured by the bounded, nonnegative, single-letter distortion measures $d_{\mathrm{d}}(\cdot, \cdot)$ and $d_{\mathrm{e}}(\cdot, \cdot)$.

We say that a nonnegative triple $\left(R, D_{\mathrm{d}}, D_{\mathrm{e}}\right)$ is achievable if for every $\epsilon>0$ and sufficiently large $n$ there exists a message set $\mathcal{M}$ of size

$$
|\mathcal{M}| \leq 2^{n(R+\epsilon)}
$$

and a triple of functions $\left(f^{(n)}, \phi^{(n)}, \psi^{(n)}\right)$ as above such that the decoder-side reconstruction constraint

$$
\frac{1}{n} \sum_{i=1}^{n} \mathrm{E}\left[d_{\mathrm{d}}\left(X_{i}, \hat{X}_{\mathrm{d}, i}\right)\right] \leq D_{\mathrm{d}}+\epsilon
$$

and the encoder-side reconstruction constraint

$$
\frac{1}{n} \sum_{i=1}^{n} \mathrm{E}\left[d_{\mathrm{e}}\left(\hat{X}_{\mathrm{d}, i}, \hat{X}_{\mathrm{e}, i}\right)\right] \leq D_{\mathrm{e}}+\epsilon
$$

are both met.

Our problem is not very interesting if the distortion constraints cannot be met even when the source sequence is revealed losslessly to the reconstructor. Consequently, we shall make the following assumption throughout:

Assumption 1: The distortion measures $d_{\mathrm{d}}$ and $d_{\mathrm{e}}$ are such that for each $x \in \mathcal{X}$ there exist $\hat{x}_{\mathrm{d}}, \hat{x}_{\mathrm{e}} \in \hat{\mathcal{X}}$ satisfying $d_{\mathrm{d}}\left(x, \hat{x}_{\mathrm{d}}\right)=0$ and $d_{\mathrm{e}}\left(\hat{x}_{\mathrm{d}}, \hat{x}_{\mathrm{e}}\right)=0$.

As we shall see, this assumption ensures that, irrespective of $D_{\mathrm{d}}, D_{\mathrm{e}} \geq 0$, the triple $\left(R, D_{\mathrm{d}}, D_{\mathrm{e}}\right)$ is achievable whenever $R \geq H(X \mid Y)$.

Given $D_{\mathrm{d}}, D_{\mathrm{e}} \geq 0$, let $\mathcal{R}\left(D_{\mathrm{d}}, D_{\mathrm{e}}\right)$ denote the set of rates $R \geq 0$ for which the tuple $\left(R, D_{\mathrm{d}}, D_{\mathrm{e}}\right)$ is achievable:

$$
\mathcal{R}\left(D_{\mathrm{d}}, D_{\mathrm{e}}\right) \triangleq\left\{R \geq 0:\left(R, D_{\mathrm{d}}, D_{\mathrm{e}}\right) \text { is achievable }\right\} .
$$

Notice that by the assumption above, the set $\mathcal{R}\left(D_{\mathrm{d}}, D_{\mathrm{e}}\right)$ contains all rates $R$ exceeding $H(X \mid Y)$ and is thus nonempty. We can now define the rate-distortions function as

$$
R\left(D_{\mathrm{d}}, D_{\mathrm{e}}\right) \triangleq \min _{R \in \mathcal{R}\left(D_{\mathrm{d}}, D_{\mathrm{e}}\right)} R,
$$

where the minimum exists because the set $\mathcal{R}\left(D_{\mathrm{d}}, D_{\mathrm{e}}\right)$ is nonempty, closed, and bounded from below by 0 . We wish to find $R\left(D_{\mathrm{d}}, D_{\mathrm{e}}\right)$.

\section{B. Related Setups}

Wyner and Ziv's classic lossy source-coding problem with side information [1] is similar to our problem except that Wyner and Ziv do not impose the encoder-side reconstruction constraint (12). Informally, our problem thus reduces to the Wyner-Ziv problem if we set $D_{\mathrm{e}}$ to infinity. Wyner and Ziv's result can be summarized as follows:

Theorem 1 (Wyner and Ziv [1]): The rate-distortion function $R_{\mathrm{WZ}}\left(D_{\mathrm{d}}\right)$ in the Wyner-Ziv setup is

$$
R_{\mathrm{WZ}}\left(D_{\mathrm{d}}\right)=\min _{Z, \phi}(I(X ; Z)-I(Y ; Z))
$$

where $(X, Y) \sim P_{X Y}$, and where the minimization is over all functions $\phi: \mathcal{Y} \times \mathcal{Z} \rightarrow \hat{\mathcal{X}}$ and discrete random variable $Z$ for which: $Z$ takes values in an auxiliary alphabet $\mathcal{Z}$ of size at $\operatorname{most}|\mathcal{X}|+1$;

$$
Z \multimap-X \multimap Y
$$

forms a Markov chain; and

$$
\mathrm{E}\left[d_{\mathrm{d}}(X, \phi(Y, Z))\right] \leq D_{\mathrm{d}} .
$$

Since imposing the encoder-side reconstruction constraint (12) cannot enlarge the set of achievable rates,

$$
R\left(D_{\mathrm{d}}, D_{\mathrm{e}}\right) \geq R_{\mathrm{WZ}}\left(D_{\mathrm{d}}\right) .
$$

Equality holds whenever the encoder-side reconstruction constraint (12) does not pinch. For example, when $\hat{\mathcal{X}}=\mathcal{X}$; $D_{\mathrm{d}}=D_{\mathrm{e}} ;$ and

$$
d_{\mathrm{e}}(\hat{x}, x)=d_{\mathrm{d}}(x, \hat{x}), \quad x, \hat{x} \in \mathcal{X} .
$$

Indeed, in this case the encoder can set $\hat{X}_{\mathrm{e}, i}$ to be $X_{i}$, which results in (12) being identical to (11) and thus superfluous.

Steinberg's setup in [2] is obtained from ours by replacing the encoder-side distortion constraint (12) by the more stringent perfect-reconstruction constraint

$$
\operatorname{Pr}\left[\hat{X}_{\mathrm{e}}^{n} \neq \hat{X}_{\mathrm{d}}^{n}\right] \leq \epsilon .
$$

Theorem 2 (Steinberg [2]): The rate-distortion function $R_{\mathrm{cr}}\left(D_{\mathrm{d}}\right)$ in Steinberg's setup is

$$
R_{\mathrm{cr}}\left(D_{\mathrm{d}}\right)=\min _{\hat{X}}(I(X ; \hat{X})-I(Y ; \hat{X})),
$$

where the minimization is over all $\hat{X}$ taking value in $\hat{\mathcal{X}}$ and satisfying

$$
\hat{X} \multimap-\circ \multimap Y
$$

and

$$
\mathrm{E}\left[d_{\mathrm{d}}(X, \hat{X})\right] \leq D_{\mathrm{d}}
$$

Remark 3: Constraint (20) is equivalent to the blockdistortion constraint

$$
\mathrm{E}\left[\mathbb{I}\left\{\hat{X}_{\mathrm{e}}^{n} \neq \hat{X}_{\mathrm{d}}^{n}\right\}\right] \leq \epsilon .
$$

Thus, when in our setup $d_{\mathrm{e}}(\cdot, \cdot)$ is the Hamming distortion and $D_{\mathrm{e}}=0$, then Steinberg's setup differs from ours only in that (20) is a block-distortion constraint whereas (12) is an average-per-symbol distortion constraint.

\section{Results}

To describe the rate-distortions function for the setup of Section II-A, we introduce the function $\tilde{R}\left(D_{\mathrm{d}}, D_{\mathrm{e}}\right)$. The expression for $\tilde{R}\left(D_{\mathrm{d}}, D_{\mathrm{e}}\right)$ is similar to the expression for $R_{\mathrm{WZ}}\left(D_{\mathrm{d}}\right)$ in (15) except that in the expression for $\tilde{R}\left(D_{\mathrm{d}}, D_{\mathrm{e}}\right)$ we have the additional constraint; see (28) ahead.

Given the joint law $P_{X Y}$ of the source and side information, and given the distortion measures $d_{\mathrm{d}}, d_{\mathrm{e}}$, this function is defined as

$$
\tilde{R}\left(D_{\mathrm{d}}, D_{\mathrm{e}}\right)=\min _{Z, \phi, \psi}(I(X ; Z)-I(Y ; Z))
$$


where the minimization is over all discrete random variables $Z$ taking value in some finite auxiliary alphabet $\mathcal{Z}$ and forming the Markov chain

$$
Z \multimap-\cap \multimap Y
$$

and over the functions $\phi: \mathcal{Y} \times \mathcal{Z} \rightarrow \hat{\mathcal{X}}$ and $\psi: \mathcal{X} \times \mathcal{Z} \rightarrow \hat{\mathcal{X}}$ satisfying

$$
\begin{aligned}
\mathrm{E}\left[d_{\mathrm{d}}(X, \phi(Y, Z))\right] & \leq D_{\mathrm{d}} \\
\mathrm{E}\left[d_{\mathrm{e}}(\phi(Y, Z), \psi(X, Z))\right] & \leq D_{\mathrm{e}}
\end{aligned}
$$

Note that, thanks to Assumption 1, the feasible set in (25) is not empty: we can choose $Z$ as $X$ and $\phi, \psi$ as the functions whose existence is guaranteed by the assumption. This choice demonstrates that

$$
\tilde{R}\left(D_{\mathrm{d}}, D_{\mathrm{e}}\right) \leq H(X \mid Y)
$$

Using the convex cover method [9] it can be shown that:

Remark 4: Allowing for sets $\mathcal{Z}$ of cardinality greater than $|\mathcal{X}|+3$ does not decrease the value of the optimization problem.

A consequence of this remark is that the minimum in (25) is achieved: indeed, we may choose $\mathcal{Z}$ as the set $\{1, \ldots,|\mathcal{X}|+3\}$ with result that there are only a finite number of functions $\phi, \psi$, and the problem is reduced to minimizing a continuous function over a compact set.

Like the Wyner-Ziv rate-distortion function $R_{\mathrm{WZ}}(\cdot)$ [10], [11], also the function $\tilde{R}\left(D_{\mathrm{d}}, D_{\mathrm{e}}\right)$ can be written in terms of Shannon strategies, but we will have no use for that.

The key properties of $\tilde{R}\left(D_{\mathrm{d}}, D_{\mathrm{e}}\right)$ are summarized in the following proposition:

Proposition 5 (Key Properties of the Function $\tilde{R}\left(D_{d}, D_{e}\right)$ ): The function $\tilde{R}\left(D_{\mathrm{d}}, D_{\mathrm{e}}\right): \mathbb{R}_{+}^{2} \rightarrow \mathbb{R}_{+}$is bounded from above by $H(X \mid Y)$ and is nondecreasing in the distortions

$$
\left(D_{\mathrm{d}}^{\prime} \geq D_{\mathrm{d}} \text { and } D_{\mathrm{e}}^{\prime} \geq D_{\mathrm{e}}\right) \Rightarrow\left(\tilde{R}\left(D_{\mathrm{d}}^{\prime}, D_{\mathrm{e}}^{\prime}\right) \leq \tilde{R}\left(D_{\mathrm{d}}, D_{\mathrm{e}}\right)\right) .
$$

Moreover, it is convex and continuous over $\mathbb{R}_{+}^{2}$.

Proof: See Appendix B.

Our main result can be now stated as:

Theorem 6: The rate-distortions function for the setup in Section II-A is equal to $\tilde{R}\left(D_{\mathrm{d}}, D_{\mathrm{e}}\right)$

$$
R\left(D_{\mathrm{d}}, D_{\mathrm{e}}\right)=\tilde{R}\left(D_{\mathrm{d}}, D_{\mathrm{e}}\right) \text {. }
$$

Proof: The coding scheme that establishes achievability is a variation on the coding scheme of Wyner and Ziv [1] and is thus only sketched. Its analysis is omitted.

Fix $Z, \phi, \psi$ satisfying (26) and (28), and fix also a blocklength $n$ and some (small) $\epsilon>0$. Let $\mathcal{C}$ be a random blocklength- $n$ codebook with $\left\lfloor 2^{n(I(X ; Z)-I(Y ; Z)+2 \epsilon)}\right\rfloor$ bins, each containing approximately $2^{n(I(Y ; Z)-\epsilon)}$ codewords with the total number of codewords thus being $\left\lfloor 2^{n(I(X ; Z)+\epsilon)}\right\rfloor$. Generate the codewords independently with the components of each codeword being drawn IID $P_{Z}$. Number the bins 1 through $\left\lfloor 2^{n(I(X ; Z)-I(Y ; Z)+2 \epsilon)}\right\rfloor$.

Upon observing the source sequence $X^{n}$, the encoder seeks a codeword $Z^{* n}$ in $\mathcal{C}$ that is jointly typical with $X^{n}$. If successful, it sends the number of the bin containing $Z^{* n}$ as the message $M$. It also produces the reconstruction sequence $\hat{X}_{\mathrm{e}}^{n}$ by applying the function $\psi$ componentwise to $Z^{* n}$ and $X^{n}$. The decoder seeks a codeword $\hat{Z}^{n}$ in Bin $M$ that is jointly typical with its side-information $Y^{n}$ and applies the reconstruction function $\phi$ componentwise to $\hat{Z}^{n}$ and $Y^{n}$ to produce $\hat{X}_{\mathrm{d}}^{n}$.

The converse is proved in Subsection II-D.

Though not identical, Steinberg's setup is very similar to our setup when $\mathrm{d}_{\mathrm{e}}(\cdot, \cdot)$ is the Hamming distortion and $D_{\mathrm{e}}$ is zero (Remark 3). It is therefore not surprising ${ }^{2}$ that, as the following corollary shows, the two setups lead to identical rates:

Corollary 7: Let $d_{\mathrm{d}}(\cdot, \cdot)$ be arbitrary, and let $d_{\mathrm{e}}(\cdot, \cdot)$ be the Hamming distortion measure

$$
d_{\mathrm{e}}\left(\hat{x}_{\mathrm{d}}, \hat{x}_{\mathrm{e}}\right)=\mathbb{I}\left\{\hat{x}_{\mathrm{d}} \neq \hat{x}_{\mathrm{e}}\right\}, \quad \hat{x}_{\mathrm{d}}, \hat{x}_{\mathrm{e}} \in \hat{\mathcal{X}} .
$$

Then

$$
\left.R\left(D_{\mathrm{d}}, D_{\mathrm{e}}\right)\right|_{D_{\mathrm{e}}=0}=R_{\mathrm{cr}}\left(D_{\mathrm{d}}\right) .
$$

Proof: See Appendix A.

Remark 8: Our results can be extended to a scenario where the encoder observes not only the source sequence $\left\{X_{i}\right\}$ but also some sequence $\left\{W_{i}\right\}$ which is correlated with the decoder's side-information sequence $\left\{Y_{i}\right\}$. This additional sequence $\left\{W_{i}\right\}$ makes it easier for the encoder to estimate the decoder's reconstruction sequence and thus allows the decoder to rely more heavily on its side information $\left\{Y_{i}\right\}$. To see how this seemingly more general scenario reduces to our scenario assume that $\left\{\left(X_{i}, W_{i}, Y_{i}\right)\right\}_{i=1}^{n}$ are IID random triples of law $P_{X W Y}$ and that $W_{i}$ takes value in the finite set $\mathcal{W}$. Consider now a new IID source $\left\{\tilde{X}_{i}\right\}$ taking value in the set $\tilde{\mathcal{X}}=\mathcal{X} \times \mathcal{W}$ according to the law $P_{X W}$ with $\tilde{X}_{i}=\left(X_{i}, W_{i}\right)$. The encoder now observes the source sequence $\left\{\tilde{X}_{i}\right\}$ only and no additional sequences. The decoder side information is still $\left\{Y_{i}\right\}$, and the joint law of $\tilde{X}_{i}, Y_{i}$ is $P_{X W Y}$. Finally define the new decoder distortion measure $\tilde{d}_{\mathrm{d}}: \tilde{\mathcal{X}} \times \hat{\mathcal{X}} \rightarrow \mathbb{R}^{+}$as

$$
\tilde{d}_{\mathrm{d}}\left(\left(X_{i}, W_{i}\right), \hat{X}_{i}\right)=d_{\mathrm{d}}\left(X_{i}, \hat{X}_{i}\right),
$$

i.e., the distortion measure $\tilde{d}_{\mathrm{d}}$ does not depend on the $W_{i}$-component. Solving the original scenario for this new source and new decoder distortion measure is equivalent to solving the seemingly more general problem we described.

These observations apply also to the plain Wyner-Ziv problem without encoder-side reconstruction constraint, see, e.g., [14].

\section{Proof of the Converse to Theorem 6}

To establish the converse, we show that if a triple $\left(R, D_{\mathrm{d}}, D_{\mathrm{e}}\right)$ is achievable, then for every $\epsilon>0$

$$
R+\epsilon \geq \tilde{R}\left(D_{\mathrm{d}}+\epsilon, D_{\mathrm{e}}+\epsilon\right) .
$$

Since $\tilde{R}\left(D_{\mathrm{d}}, D_{\mathrm{e}}\right)$ is continuous (Proposition 5), and since $\epsilon$ can be arbitrarily small, this implies that $R \geq \tilde{R}\left(D_{\mathrm{d}}, D_{\mathrm{e}}\right)$ whenever $\left(R, D_{\mathrm{d}}, D_{\mathrm{e}}\right)$ is achievable, and consequently that $R\left(D_{\mathrm{d}}, D_{\mathrm{e}}\right) \geq$ $\tilde{R}\left(D_{\mathrm{d}}, D_{\mathrm{e}}\right)$.

The first part of our proof identifying the auxiliary random variable $Z_{i}$ (44) and the function $\phi_{i}(46)$ is similar to the proof

\footnotetext{
${ }^{2}$ See [9, Section 3.6.4], [12], and [13] for related discussions.
} 
of the Wyner-Ziv result [9]. For a given blocklength- $n$ code $f^{(n)}, \phi^{(n)}, \psi^{(n)}$ satisfying (10)-(12), we have

$$
\begin{aligned}
n(R+\epsilon) & \stackrel{(\mathrm{a})}{\geq} H(M) \\
& \stackrel{\text { (b) }}{\geq} I\left(X^{n} ; M \mid Y^{n}\right) \\
& \stackrel{(\mathrm{c})}{=} \sum_{i=1}^{n} I\left(X_{i} ; M \mid Y^{n}, X^{i-1}\right) \\
& =\sum_{i=1}^{n} H\left(X_{i} \mid Y^{n}, X^{i-1}\right)-H\left(X_{i} \mid M, Y^{n}, X^{i-1}\right) \\
& \stackrel{(\mathrm{d})}{=} \sum_{i=1}^{n} H\left(X_{i} \mid Y_{i}\right)-H\left(X_{i} \mid M, Y^{n}, X^{i-1}\right) \\
& \stackrel{(\mathrm{e})}{\geq} \sum_{i=1}^{n} H\left(X_{i} \mid Y_{i}\right)-H\left(X_{i} \mid M, Y^{n}\right) \\
& \stackrel{(\mathrm{f})}{=} \sum_{i=1}^{n} H\left(X_{i} \mid Y_{i}\right)-H\left(X_{i} \mid Z_{i}, Y_{i}\right) \\
& =\sum_{i=1}^{n} I\left(X_{i} ; Z_{i} \mid Y_{i}\right) \\
& \stackrel{(\mathrm{g})}{=} \sum_{i=1}^{n} H\left(Z_{i} \mid Y_{i}\right)-H\left(Z_{i} \mid X_{i}\right) \\
& =\sum_{i=1}^{n} I\left(X_{i} ; Z_{i}\right)-I\left(Y_{i} ; Z_{i}\right),
\end{aligned}
$$

where (a) follows by (10); (b) follows because conditioning cannot increase entropy and because $H\left(M \mid Y^{n}, X^{n}\right) \geq 0$; (c) follows from the chain rule for mutual information; (d) follows because the pair $X_{i}, Y_{i}$ is independent of the tuple $\left(X_{1}^{i-1}, Y_{1}^{i-1}, Y_{i+1}^{n}\right)$; (e) follows from the fact that conditioning cannot increase entropy; (f) follows by defining

$$
Z_{i} \triangleq\left(M, Y^{i-1}, Y_{i+1}^{n}\right) ;
$$

and $(\mathrm{g})$ follows because with the definition above

$$
Z_{i} \multimap-X_{i} \multimap Y_{i} .
$$

Denote by $\phi_{i}^{(n)}$ the function that maps $\left(M, Y^{n}\right)$ to the $i$-th component of the $n$-tuple $\phi^{(n)}\left(M, Y^{n}\right)$, and denote by $\psi_{i}^{(n)}$ the function that maps $X^{n}$ to the $i$-th component of the $n$-tuple $\psi^{(n)}\left(X^{n}\right)$. Since there is a one-to-one correspondence between the pairs $\left(Y_{i}, Z_{i}\right)$ and $\left(M, Y^{n}\right)$, we can define a function $\phi_{i}$ that maps $\left(Y_{i}, Z_{i}\right)$ to $\phi_{i}^{(n)}\left(M, Y^{n}\right)$

$$
\phi_{i}\left(Y_{i}, Z_{i}\right) \triangleq \phi_{i}^{(n)}\left(M, Y^{n}\right) \text {. }
$$

We now define

$$
D_{\mathrm{d}, i} \triangleq \mathrm{E}\left[d_{\mathrm{d}}\left(X_{i}, \phi_{i}^{(n)}\left(M, Y^{n}\right)\right)\right],
$$

where $\mathrm{E}[\cdot]$ is with respect to $P_{X^{n} Y^{n}}$. By definitions (46) and (47),

$$
\mathrm{E}\left[d_{\mathrm{d}}\left(X_{i}, \phi_{i}\left(Y_{i}, Z_{i}\right)\right)\right]=D_{\mathrm{d}, i},
$$

where E[·] is with respect to $P_{X_{i} Y_{i}} P_{Z_{i} \mid X_{i}}$.

We next turn to the encoder-side distortion. We will show that there exists a deterministic function $\psi_{i}: \mathcal{X} \times \mathcal{Z} \rightarrow \hat{\mathcal{X}}$ that achieves a distortion no larger than $D_{\mathrm{e}, i}$, where $D_{\mathrm{e}, i}$ is the distortion achieved by $\psi_{i}^{(n)}\left(X^{n}\right)$, namely,

$$
D_{\mathrm{e}, i} \triangleq \mathrm{E}\left[d_{\mathrm{e}}\left(\phi_{i}^{(n)}\left(M, Y^{n}\right), \psi_{i}^{(n)}\left(X^{n}\right)\right)\right] .
$$

This is the key step in our converse proof. It is similar to steps applied in [15].

To this end, we express $D_{\mathrm{e}, i}$ as

$$
\begin{aligned}
D_{\mathrm{e}, i} & =\mathrm{E}_{X^{n}, Y_{i}, Z_{i}}\left[d_{\mathrm{e}}\left(\phi_{i}\left(Y_{i}, Z_{i}\right), \psi_{i}^{(n)}\left(X^{n}\right)\right)\right] \\
& =\mathrm{E}_{X^{n}, Z_{i}} \mathrm{E}_{Y_{i} \mid X^{n}, Z_{i}}\left[d_{\mathrm{e}}\left(\phi_{i}\left(Y_{i}, Z_{i}\right), \psi_{i}^{(n)}\left(X^{n}\right)\right)\right] \\
& =\mathrm{E}_{X^{n}, Z_{i}} \mathrm{E}_{Y_{i} \mid X_{i}, X_{i}, Z_{i}}\left[d_{\mathrm{e}}\left(\phi_{i}\left(Y_{i}, Z_{i}\right), \psi_{i}^{(n)}\left(X_{i}, X_{i}\right)\right)\right],
\end{aligned}
$$

where $X_{\backslash i} \triangleq\left(X^{i-1}, X_{i+1}^{n}\right)$. For every $\left(x_{i}, z_{i}\right) \in \mathcal{X} \times \mathcal{Z}$, we define $x_{i i}^{*}\left(x_{i}, z_{i}\right)$ (or for short $\left.x_{i i}^{*}\right)$ as ${ }^{3}$ :

$$
\begin{aligned}
x_{i}^{*}\left(x_{i}, z_{i}\right) & \triangleq \underset{x_{i} \in \mathcal{X}^{n-1}}{\arg \min } \\
\mathrm{E}_{Y_{i} \mid X_{i}=x_{i}, X_{i}=x_{i}, Z_{i}=z_{i}} & {\left[d_{\mathrm{e}}\left(\phi_{i}\left(Y_{i}, z_{i}\right), \psi_{i}^{(n)}\left(x_{i}, x_{i}\right)\right)\right] }
\end{aligned}
$$

or in any other way that guarantees

$$
\begin{aligned}
& \mathrm{E}_{X_{\mid} \mid X_{i}=x_{i}, Z_{i}=z_{i}} \\
& \quad \mathrm{E}_{Y_{i} \mid X_{i}=x_{i}, X_{i}, Z_{i}=z_{i}}\left[d_{\mathrm{e}}\left(\phi_{i}\left(Y_{i}, z_{i}\right), \psi_{i}^{(n)}\left(x_{i}, X_{\mid i}\right)\right)\right] \\
& \quad \geq \mathrm{E}_{Y_{i} \mid X_{i}=x_{i}, X_{i}=x_{i}^{*}, Z_{i}=z_{i}}\left[d_{\mathrm{e}}\left(\phi_{i}\left(Y_{i}, z_{i}\right), \psi_{i}^{(n)}\left(x_{i}, x_{i}^{*}\right)\right)\right] .
\end{aligned}
$$

We can now define the function $\psi_{i}$ as

$$
\begin{aligned}
\psi_{i}: \mathcal{X} \times \mathcal{Z} & \rightarrow \hat{\mathcal{X}} \\
\left(x_{i}, z_{i}\right) & \mapsto \psi_{i}^{(n)}\left(x_{i}, x_{i}^{*}\left(x_{i}, z_{i}\right)\right) .
\end{aligned}
$$

For every $\left(x_{i}, x_{i}, z_{i}\right) \in \mathcal{X}^{n} \times \mathcal{Z}$, we have

$$
\begin{aligned}
& \mathrm{E}_{Y_{i} \mid X_{i}=x_{i}, X_{i}=x_{i}, Z_{i}=z_{i}}\left[d_{\mathrm{e}}\left(\phi_{i}\left(Y_{i}, z_{i}\right), \psi_{i}^{(n)}\left(x_{i}, x_{i}\right)\right)\right] \\
& \stackrel{(a)}{\geq} \mathrm{E}_{Y_{i} \mid X_{i}=x_{i}, X_{i}=x_{i}^{*}, Z_{i}=z_{i}}\left[d_{\mathrm{e}}\left(\phi_{i}\left(Y_{i}, z_{i}\right), \psi_{i}^{(n)}\left(x_{i}, x_{i}^{*}\right)\right)\right] \\
& \stackrel{(\text { b) }}{=} \mathrm{E}_{Y_{i} \mid X_{i}=x_{i}, Z_{i}=z_{i}}\left[d_{\mathrm{e}}\left(\phi_{i}\left(Y_{i}, z_{i}\right), \psi_{i}^{(n)}\left(x_{i}, x_{i}^{*}\right)\right)\right] \\
& \stackrel{(\text { c) }}{=} \mathrm{E}_{Y_{i} \mid X_{i}=x_{i}, Z_{i}=z_{i}}\left[d_{\mathrm{e}}\left(\phi_{i}\left(Y_{i}, z_{i}\right), \psi_{i}\left(x_{i}, z_{i}\right)\right)\right],
\end{aligned}
$$

where (a) follows from the definition of $x_{i}^{*}$; (b) follows because

$$
X_{i} \multimap-\left(X_{i}, Z_{i}\right) \multimap Y_{i} ;
$$

and (c) follows from the definition of $\psi_{i}$ (55).

It now follows from (52) and (58) that

$$
\mathrm{E}_{X_{i}, Y_{i}, Z_{i}}\left[d_{\mathrm{e}}\left(\phi_{i}\left(Y_{i}, Z_{i}\right), \psi_{i}\left(X_{i}, Z_{i}\right)\right)\right] \leq D_{\mathrm{e}, i} .
$$

Continuing from (43) we thus obtain

$$
n(R+\epsilon) \geq \sum_{i=1}^{n} I\left(X_{i} ; Z_{i}\right)-I\left(Y_{i} ; Z_{i}\right)
$$

${ }^{3}$ If $\arg \min$ is not unique, $x_{i}\left(x_{i}, z_{i}\right)$ is defined as the first in lexicographical order. 


$$
\begin{aligned}
& \stackrel{\text { (a) }}{\geq} \sum_{i=1}^{n} \tilde{R}\left(D_{\mathrm{d}, i}, D_{\mathrm{e}, i}\right) \\
& \stackrel{\text { (b) }}{=} n \frac{1}{n} \sum_{i=1}^{n} \tilde{R}\left(D_{\mathrm{d}, i}, D_{\mathrm{e}, i}\right) \\
& \stackrel{\text { (c) }}{\geq} n \tilde{R}\left(\frac{1}{n} \sum_{i=1}^{n} D_{\mathrm{d}, i}, \frac{1}{n} \sum_{i=1}^{n} D_{\mathrm{e}, i}\right) \\
& \stackrel{\text { (d) }}{\geq} n \tilde{R}\left(D_{\mathrm{d}}+\epsilon, D_{\mathrm{e}}+\epsilon\right)
\end{aligned}
$$

where (a) follows from the definition of $\tilde{R}\left(D_{\mathrm{d}}, D_{\mathrm{e}}\right)$ and from (45), (48), and (60); (b) follows by multiplying by 1 ; (c) follows from the convexity of $\tilde{R}\left(D_{\mathrm{d}}, D_{\mathrm{e}}\right)$ (Proposition 5); and (d) follows from the monotonicity of $\tilde{R}\left(D_{\mathrm{d}}, D_{\mathrm{e}}\right)$ (Proposition 5) and the fact that $\frac{1}{n} \sum_{i=1}^{n} D_{\mathrm{d}, i} \leq D_{\mathrm{d}}+\epsilon$ and $\frac{1}{n} \sum_{i=1}^{n} D_{\mathrm{e}, i} \leq D_{\mathrm{e}}+\epsilon$. This establishes (33) and thus concludes the proof of the converse.

\section{Gaussian SOURCE AND QuAdRATIC DistorTIONS}

\section{A. Setup}

We next consider the case where the source, side information, and reconstruction alphabets $\mathcal{X}, \mathcal{Y}, \hat{\mathcal{X}}$ are the reals $\mathbb{R}$; the distortion functions $d_{\mathrm{d}}$ and $d_{\mathrm{e}}$ are quadratic

$$
\begin{aligned}
d_{\mathrm{d}}\left(x, \hat{x}_{\mathrm{d}}\right) & =\left(x-\hat{x}_{\mathrm{d}}\right)^{2}, \\
d_{\mathrm{e}}\left(\hat{x}_{\mathrm{d}}, \hat{x}_{\mathrm{e}}\right) & =\left(\hat{x}_{\mathrm{d}}-\hat{x}_{\mathrm{e}}\right)^{2} ;
\end{aligned}
$$

and the source and side-information pair $(X, Y)$ is a centered bivariate Gaussian, where $X$ is of positive variance $\sigma_{X}^{2}$

$$
\sigma_{X}>0
$$

and where $Y=\xi X+U$ for some centered variance- $\sigma_{U}^{2}$ Gaussian $U$ that is independent of $X$ for some nonzero constant $\xi{ }^{4}$ The rate-distortions function depends on $\xi$ only through the ratio $\sigma_{U}^{2} / \xi^{2}$, because the receiver can premultiply its side information by $\xi^{-1}$ without affecting the ratedistortions function. In the following we thus assume that $\xi=1$, i.e.,

$$
Y=X+U
$$

We denote the rate-distortions function for this setup by $R^{\mathrm{G}}\left(D_{\mathrm{d}}, D_{\mathrm{e}}\right)$.

When $\sigma_{U}$ is zero the problem is not interesting, because in this case the source sequence is determined by the side information, and $R^{\mathrm{G}}\left(D_{\mathrm{d}}, D_{\mathrm{e}}\right)$ is thus zero for all nonnegative values of $D_{\mathrm{d}}$ and $D_{\mathrm{e}}$. We shall henceforth thus assume

$$
\sigma_{U}>0
$$

In this case, no finite rate can allow $D_{\mathrm{d}}$ to be zero (even if we ignore the encoder-side reconstruction constraint). Thus, we shall also assume

$$
D_{\mathrm{d}}>0
$$

\footnotetext{
${ }^{4}$ The problem is not interesting when $\xi$ is zero, because in this case the side information is independent of the source and is thus irrelevant.
}

\section{B. Related Work}

As we have seen in Section II-B, the Wyner-Ziv setup is obtained from ours if the encoder-side reconstruction constraint (12) is omitted, and Steinberg's common reconstruction setup is obtained if (12) is replaced by (20).

For a Gaussian source and quadratic distortion measures, Steinberg's common reconstruction rate-distortion function is [2]

$$
R_{\mathrm{cr}}^{\mathrm{G}}\left(D_{\mathrm{d}}\right)=\frac{1}{2} \log ^{+} \frac{\sigma_{X}^{2}\left(\sigma_{U}^{2}+D_{\mathrm{d}}\right)}{\left(\sigma_{X}^{2}+\sigma_{U}^{2}\right) D_{\mathrm{d}}},
$$

and the Wyner-Ziv rate-distortion function is [1]

$$
R_{\mathrm{WZ}}^{\mathrm{G}}\left(D_{\mathrm{d}}\right)=\frac{1}{2} \log ^{+} \frac{\sigma_{X}^{2} \sigma_{U}^{2}}{\left(\sigma_{X}^{2}+\sigma_{U}^{2}\right) D_{\mathrm{d}}} .
$$

Note that (73) is also the rate-distortion function when the side information is revealed not only to the decoder but also to the encoder.

\section{Result}

Theorem 9: For a Gaussian source and quadratic distortion measures, the rate-distortions function $R^{\mathrm{G}}\left(D_{\mathrm{d}}, D_{\mathrm{e}}\right)$ can be expressed as follows:

$$
\begin{aligned}
& \text { If } \sqrt{D_{\mathrm{e}} \sigma_{U}^{2}} \geq \min \left\{D_{\mathrm{d}}, \frac{\sigma_{X}^{2} \sigma_{U}^{2}}{\sigma_{X}^{2}+\sigma_{U}^{2}}\right\} \text {, then } \\
& R^{\mathrm{G}}\left(D_{\mathrm{d}}, D_{\mathrm{e}}\right)=\frac{1}{2} \log ^{+} \frac{\sigma_{X}^{2} \sigma_{U}^{2}}{\left(\sigma_{X}^{2}+\sigma_{U}^{2}\right) D_{\mathrm{d}}} . \\
& \text { If } \sqrt{D_{\mathrm{e}} \sigma_{U}^{2}}<\min \left\{D_{\mathrm{d}}, \frac{\sigma_{X}^{2} \sigma_{U}^{2}}{\sigma_{X}^{2}+\sigma_{U}^{2}}\right\} \text {, then } \\
& R^{\mathrm{G}}\left(D_{\mathrm{d}}, D_{\mathrm{e}}\right)=\frac{1}{2} \log ^{+}\left(\frac{\sigma_{X}^{2}}{\sigma_{X}^{2}+\sigma_{U}^{2}} \frac{\sigma_{U}^{2}+D_{\mathrm{d}}-2 \sqrt{\sigma_{U}^{2} D_{\mathrm{e}}}}{D_{\mathrm{d}}-D_{\mathrm{e}}}\right) .
\end{aligned}
$$

Proof: The direct part is proved in Section III-D and the converse in Section III-E.

Remark 10: If $D_{\mathrm{e}}=0$, then our rate-distortions function $R^{\mathrm{G}}\left(D_{\mathrm{d}}, 0\right)$ coincides with Steinberg's common-reconstruction rate-distortion function $R_{\mathrm{cr}}^{\mathrm{G}}\left(D_{\mathrm{d}}\right)$ of (72):

$$
\left.R^{\mathrm{G}}\left(D_{\mathrm{d}}, D_{\mathrm{e}}\right)\right|_{D_{\mathrm{e}}=0}=R_{\mathrm{cr}}^{\mathrm{G}}\left(D_{\mathrm{d}}\right) \text {. }
$$

If $D_{\mathrm{d}}$ and $D_{\mathrm{e}}$ are such that

$$
\sqrt{D_{\mathrm{e}} \sigma_{U}^{2}} \geq \min \left\{D_{\mathrm{d}}, \frac{\sigma_{X}^{2} \sigma_{U}^{2}}{\sigma_{X}^{2}+\sigma_{U}^{2}}\right\}
$$

or

$$
\frac{\sigma_{X}^{2}+\sigma_{U}^{2}}{\sigma_{X}^{2}} \geq \frac{\sigma_{U}^{2}+D_{\mathrm{d}}-2 \sqrt{\sigma_{U}^{2} D_{\mathrm{e}}}}{D_{\mathrm{d}}-D_{\mathrm{e}}}
$$

then $R^{\mathrm{G}}\left(D_{\mathrm{d}}, D_{\mathrm{e}}\right)$ coincides with the Wyner-Ziv rate-distortion function $R_{\mathrm{WZ}}^{\mathrm{G}}\left(D_{\mathrm{d}}\right)$ in (73). Thus, if (77) or (78) holds, then relaxing Constraint (12) and/or revealing the side information also to the encoder does not decrease the rate-distortions function. 
The reasons for this are as follows. Under condition (77) it is possible to find some $\hat{X}_{\mathrm{e}}^{n}$ that satisfies the encoderside reconstruction constraint (12) when $\hat{X}_{\mathrm{d}}^{n}$ is chosen as the optimum reconstruction for the Wyner-Ziv problem. (For more details see the direct part described in the next section.) Under condition (78), by Theorem $9, R^{\mathrm{G}}\left(D_{\mathrm{d}}, D_{\mathrm{e}}\right)=0$. Since the required rate for the relaxed Wyner-Ziv problem is nonnegative and no larger than $R^{\mathrm{G}}\left(D_{\mathrm{d}}, D_{\mathrm{e}}\right)=0$, we conclude that in this case also $R_{\mathrm{WZ}}^{\mathrm{G}}\left(D_{\mathrm{d}}\right)=0$.

\section{The Direct Part of Theorem 9}

In the two cases that we shall describe in (79) and (82) ahead, no encoding is necessary because the encoder and the decoder can produce sufficiently good reconstructions $\hat{X}_{\mathrm{e}}^{n}$ and $\hat{X}_{\mathrm{d}}^{n}$ based solely on their observed sequences $X^{n}$ and $Y^{n}$. In these cases $R^{\mathrm{G}}\left(D_{\mathrm{d}}, D_{\mathrm{e}}\right)$ is thus zero.

1) If

$$
\sqrt{D_{\mathrm{e}} \sigma_{U}^{2}} \geq \min \left\{D_{\mathrm{d}}, \frac{\sigma_{X}^{2} \sigma_{U}^{2}}{\sigma_{X}^{2}+\sigma_{U}^{2}}\right\}
$$

and

$$
D_{\mathrm{d}} \geq \frac{\sigma_{X}^{2} \sigma_{U}^{2}}{\sigma_{X}^{2}+\sigma_{U}^{2}},
$$

then the encoder and decoder can produce the sequences

$$
\begin{aligned}
& \hat{X}_{\mathrm{e}}^{n}=\frac{\sigma_{X}^{2}}{\sigma_{X}^{2}+\sigma_{U}^{2}} X^{n} \\
& \hat{X}_{\mathrm{d}}^{n}=\frac{\sigma_{X}^{2}}{\sigma_{X}^{2}+\sigma_{U}^{2}} Y^{n}
\end{aligned}
$$

which satisfy the distortion constraints.

2) If

$$
\sqrt{D_{\mathrm{e}} \sigma_{U}^{2}}<\min \left\{D_{\mathrm{d}}, \frac{\sigma_{X}^{2} \sigma_{U}^{2}}{\sigma_{X}^{2}+\sigma_{U}^{2}}\right\}
$$

and

$$
D_{\mathrm{d}} \geq \sigma_{X}^{2}\left(1-\sqrt{\frac{D_{\mathrm{e}}}{\sigma_{U}^{2}}}\right)^{2}+D_{\mathrm{e}},
$$

then the encoder and decoder can produce the sequences

$$
\begin{aligned}
& \hat{X}_{\mathrm{e}}^{n}=\sqrt{\frac{D_{\mathrm{e}}}{\sigma_{U}^{2}}} X^{n} \\
& \hat{X}_{\mathrm{d}}^{n}=\sqrt{\frac{D_{\mathrm{e}}}{\sigma_{U}^{2}}} Y^{n}
\end{aligned}
$$

which satisfy the distortion constraints.

The achievability of Theorem 9 in the remaining cases will be established using the following proposition with a judicious choice of the parameters.

Proposition 11: For the setup in Section III-A of a Gaussian source and quadratic distortion measures, the tuple $\left(R, D_{\mathrm{d}}, D_{\mathrm{e}}\right)$ is achievable whenever

$$
R \geq \frac{1}{2} \log \frac{\sigma_{X}^{2} \sigma_{U}^{2}+\sigma_{X}^{2} \sigma_{W}^{2}+\sigma_{U}^{2} \sigma_{W}^{2}}{\left(\sigma_{X}^{2}+\sigma_{U}^{2}\right) \sigma_{W}^{2}}
$$

for some parameters $\sigma_{W}^{2}, a>0$ and $b \geq 0$ simultaneously satisfying

$$
(1-a-b)^{2} \sigma_{X}^{2}+a^{2} \sigma_{W}^{2}+b^{2} \sigma_{U}^{2} \leq D_{\mathrm{d}}
$$

and

$$
b^{2} \sigma_{U}^{2} \leq D_{\mathrm{e}}
$$

Thus,

$$
R^{\mathrm{G}}\left(D_{\mathrm{d}}, D_{\mathrm{e}}\right) \leq \min _{a, b, \sigma_{W}^{2}} \frac{1}{2} \log \frac{\sigma_{X}^{2} \sigma_{U}^{2}+\sigma_{X}^{2} \sigma_{W}^{2}+\sigma_{U}^{2} \sigma_{W}^{2}}{\left(\sigma_{X}^{2}+\sigma_{U}^{2}\right) \sigma_{W}^{2}},
$$

where the minimization is over all $\sigma_{W}^{2}, a>0$ and $b \geq 0$ satisfying (86).

Proof: See Appendix C.

We can now prove the achievability part of Theorem 9 for the remaining cases.

3) If

$$
\sqrt{D_{\mathrm{e}} \sigma_{U}^{2}} \geq \min \left\{D_{\mathrm{d}}, \frac{\sigma_{X}^{2} \sigma_{U}^{2}}{\sigma_{X}^{2}+\sigma_{U}^{2}}\right\}
$$

and

$$
D_{\mathrm{d}}<\frac{\sigma_{X}^{2} \sigma_{U}^{2}}{\sigma_{X}^{2}+\sigma_{U}^{2}}
$$

then the choice

$$
\sigma_{W}^{2}=\frac{D_{\mathrm{d}}}{1-\frac{\sigma_{X}^{2}+\sigma_{U}^{2}}{\sigma_{X}^{2} \sigma_{U}^{2}} D_{\mathrm{d}}}
$$

(which is positive by (88b)) and

$$
\begin{aligned}
& a=\frac{D_{\mathrm{d}}}{\sigma_{W}^{2}}=1-\frac{\sigma_{X}^{2}+\sigma_{U}^{2}}{\sigma_{X}^{2} \sigma_{U}^{2}} D_{\mathrm{d}}, \\
& b=\frac{\sigma_{X}^{2}}{\sigma_{X}^{2}+\sigma_{U}^{2}}(1-a)=\frac{D_{\mathrm{d}}}{\sigma_{U}^{2}} .
\end{aligned}
$$

satisfies (86) because

$$
\begin{aligned}
(1- & a-b)^{2} \sigma_{X}^{2}+a^{2} \sigma_{W}^{2}+b^{2} \sigma_{U}^{2} \\
& =\left(\frac{\sigma_{X}^{2}+\sigma_{U}^{2}}{\sigma_{X}^{2}} b-b\right)^{2} \sigma_{X}^{2}+\frac{D_{\mathrm{d}}^{2}}{\sigma_{W}^{2}}+\frac{D_{\mathrm{d}}^{2}}{\sigma_{U}^{2}} \\
& =\frac{D_{\mathrm{d}}^{2}}{\sigma_{X}^{2}}+D_{\mathrm{d}}\left(1-\frac{\sigma_{X}^{2}+\sigma_{U}^{2}}{\sigma_{X}^{2} \sigma_{U}^{2}} D_{\mathrm{d}}\right)+\frac{D_{\mathrm{d}}^{2}}{\sigma_{U}^{2}} \\
& =D_{\mathrm{d}}
\end{aligned}
$$

and

$$
b^{2} \sigma_{U}^{2}=\frac{D_{\mathrm{d}}^{2}}{\sigma_{U}^{2}} \leq D_{\mathrm{e}} .
$$

Moreover, for this choice,

$$
\begin{gathered}
\frac{1}{2} \log \frac{\sigma_{X}^{2} \sigma_{U}^{2}+\sigma_{X}^{2} \sigma_{W}^{2}+\sigma_{U}^{2} \sigma_{W}^{2}}{\left(\sigma_{X}^{2}+\sigma_{U}^{2}\right) \sigma_{W}^{2}} \\
=\frac{1}{2} \log \frac{\sigma_{X}^{2} \sigma_{U}^{2}}{\left(\sigma_{X}^{2}+\sigma_{U}^{2}\right) D_{\mathrm{d}}} .
\end{gathered}
$$


Thus, by (92)-(94) and by Proposition 11, we conclude that when $D_{\mathrm{d}}$ and $D_{\mathrm{e}}$ satisfy (88),

$$
R^{\mathrm{G}}\left(D_{\mathrm{d}}, D_{\mathrm{e}}\right) \leq \frac{1}{2} \log \frac{\sigma_{X}^{2} \sigma_{U}^{2}}{\left(\sigma_{X}^{2}+\sigma_{U}^{2}\right) D_{\mathrm{d}}}
$$

4) If

$$
\sqrt{D_{\mathrm{e}} \sigma_{U}^{2}}<\min \left\{D_{\mathrm{d}}, \frac{\sigma_{X}^{2} \sigma_{U}^{2}}{\sigma_{X}^{2}+\sigma_{U}^{2}}\right\}
$$

and

$$
D_{\mathrm{d}}<\sigma_{X}^{2}\left(1-\sqrt{\frac{D_{\mathrm{e}}}{\sigma_{U}^{2}}}\right)^{2}+D_{\mathrm{e}},
$$

then we consider the choice

$$
\begin{aligned}
b & =\sqrt{\frac{D_{\mathrm{e}}}{\sigma_{U}^{2}}}, \\
a & =\frac{\sigma_{X}^{2}}{\sigma_{X}^{2}+\sigma_{W}^{2}}(1-b), \\
\sigma_{W}^{2} & =\frac{\sigma_{X}^{2}\left(D_{\mathrm{d}}-b^{2} \sigma_{U}^{2}\right)}{\sigma_{X}^{2}(1-b)^{2}+b^{2} \sigma_{U}^{2}-D_{\mathrm{d}}} \\
& =\frac{\sigma_{X}^{2}\left(D_{\mathrm{d}}-D_{\mathrm{e}}\right)}{\sigma_{X}^{2}\left(1-\sqrt{\frac{D_{\mathrm{e}}}{\sigma_{U}^{2}}}\right)^{2}+D_{\mathrm{e}}-D_{\mathrm{d}}} .
\end{aligned}
$$

To see that the RHS of (97c) is positive note that (96b) implies that the denominator is positive, and (96a) implies that the numerator is positive because

$$
\begin{aligned}
& \left(\sqrt{D_{\mathrm{e}} \sigma_{U}^{2}}<\min \left\{D_{\mathrm{d}}, \frac{\sigma_{X}^{2} \sigma_{U}^{2}}{\sigma_{X}^{2}+\sigma_{U}^{2}}\right\}\right) \\
& \Longrightarrow\left(D_{\mathrm{e}}<\min \left\{\sigma_{U}^{2}, D_{\mathrm{d}}\right\}\right) \text {. }
\end{aligned}
$$

(Since $\sigma_{X}^{2} /\left(\sigma_{X}^{2}+\sigma_{U}^{2}\right)$ is smaller than one, the LHS of (98) implies that $D_{\mathrm{e}}<\sigma_{U}^{2}$. This, and the fact that the LHS of (98) also implies that $D_{\mathrm{e}} \sigma_{U}^{2}<D_{\mathrm{d}}^{2}$ demonstrates that the LHS of (98) also implies that $D_{\mathrm{e}}<D_{\mathrm{d}}$.) This choice satisfies (86) because

$$
\begin{aligned}
(1 & -a-b)^{2} \sigma_{X}^{2}+a^{2} \sigma_{W}^{2}+b^{2} \sigma_{U}^{2} \\
& =\left(\frac{\sigma_{W}^{2}(1-b)}{\sigma_{X}^{2}+\sigma_{W}^{2}}\right)^{2} \sigma_{X}^{2}+\left(\frac{\sigma_{X}^{2}(1-b)}{\sigma_{X}^{2}+\sigma_{W}^{2}}\right)^{2} \sigma_{W}^{2}+D_{\mathrm{e}} \\
& =\frac{\sigma_{X}^{2}(1-b)^{2}}{\frac{\sigma_{X}^{2}}{\sigma_{W}^{2}}+1}+D_{\mathrm{e}} \\
& =\frac{\sigma_{X}^{2}(1-b)^{2}\left(D_{\mathrm{d}}-b^{2} \sigma_{U}^{2}\right)}{\sigma_{X}^{2}(1-b)^{2}}+D_{\mathrm{e}} \\
& =D_{\mathrm{d}} .
\end{aligned}
$$

and

$$
b^{2} \sigma_{U}^{2}=D_{\mathrm{e}}
$$

Moreover, for this choice,

$$
\begin{aligned}
\frac{1}{2} \log & \frac{\sigma_{X}^{2} \sigma_{U}^{2}+\sigma_{X}^{2} \sigma_{W}^{2}+\sigma_{U}^{2} \sigma_{W}^{2}}{\left(\sigma_{X}^{2}+\sigma_{U}^{2}\right) \sigma_{W}^{2}} \\
= & \frac{1}{2} \log \frac{\sigma_{X}^{2}\left(\sigma_{U}^{2}+D_{\mathrm{d}}-2 \sqrt{\sigma_{U}^{2} D_{\mathrm{e}}}\right)}{\left(\sigma_{X}^{2}+\sigma_{U}^{2}\right)\left(D_{\mathrm{d}}-D_{\mathrm{e}}\right)} .
\end{aligned}
$$

Thus, by (102)-(104) and by Proposition 11, we conclude that when (96) holds,

$$
R^{\mathrm{G}}\left(D_{\mathrm{d}}, D_{\mathrm{e}}\right) \leq \frac{1}{2} \log \frac{\sigma_{X}^{2}\left(\sigma_{U}^{2}+D_{\mathrm{d}}-2 \sqrt{\sigma_{U}^{2} D_{\mathrm{e}}}\right)}{\left(\sigma_{X}^{2}+\sigma_{U}^{2}\right)\left(D_{\mathrm{d}}-D_{\mathrm{e}}\right)} .
$$

Remark 12: The expressions in Proposition 11 and their relation to (25) become more transparent when we define

$$
\begin{aligned}
Z & =a(X+W) \\
\hat{X}_{\mathrm{d}} & =b Y+Z \\
\hat{X}_{\mathrm{e}} & =b X+Z
\end{aligned}
$$

for $a>0, b \geq 0$, and $W$ a centered Gaussian of positive variance $\sigma_{W}^{2}$ independent of the pair $(X, Y)$. With these definitions

$$
\begin{aligned}
I(X ; Z \mid Y) & =\frac{1}{2} \log \frac{\sigma_{X}^{2} \sigma_{U}^{2}+\sigma_{X}^{2} \sigma_{W}^{2}+\sigma_{U}^{2} \sigma_{W}^{2}}{\left(\sigma_{X}^{2}+\sigma_{U}^{2}\right) \sigma_{W}^{2}} \\
\mathrm{E}\left[\left(X-\hat{X}_{\mathrm{d}}\right)^{2}\right] & =(1-a-b)^{2} \sigma_{X}^{2}+a^{2} \sigma_{W}^{2}+b^{2} \sigma_{U}^{2} \\
\mathrm{E}\left[\left(\hat{X}_{\mathrm{d}}-\hat{X}_{\mathrm{e}}\right)^{2}\right] & =b^{2} \sigma_{U}^{2} .
\end{aligned}
$$

Since $Z \multimap-X \multimap-Y$ for all choices of the parameters $a>0$, $b \geq 0, \sigma_{W}^{2}>0$, we can also rewrite (87) as:

$$
R^{\mathrm{G}}\left(D_{\mathrm{d}}, D_{\mathrm{e}}\right) \leq \min _{Z, \hat{X}_{\mathrm{d}}, \hat{X}_{\mathrm{e}}} I(X ; Z \mid Y)
$$

where the minimum is over all $Z, \hat{X}_{\mathrm{d}}, \hat{X}_{\mathrm{e}}$ that are of the form in (106) and satisfy the distortion constraints

$$
\begin{aligned}
\mathrm{E}\left[\left(X-\hat{X}_{\mathrm{d}}\right)^{2}\right] & \leq D_{\mathrm{d}}, \\
\mathrm{E}\left[\left(\hat{X}_{\mathrm{d}}-\hat{X}_{\mathrm{e}}\right)^{2}\right] & \leq D_{\mathrm{e}} .
\end{aligned}
$$

\section{E. The Converse for Theorem 9}

If

$$
\sqrt{D_{\mathrm{e}} \sigma_{U}^{2}} \geq \min \left\{D_{\mathrm{d}}, \frac{\sigma_{X}^{2} \sigma_{U}^{2}}{\sigma_{X}^{2}+\sigma_{U}^{2}}\right\}
$$

then the converse follows by relaxing the constraint (12); see Remark 10. We thus focus on the case where

$$
\sqrt{D_{\mathrm{e}} \sigma_{U}^{2}}<\min \left\{D_{\mathrm{d}}, \frac{\sigma_{X}^{2} \sigma_{U}^{2}}{\sigma_{X}^{2}+\sigma_{U}^{2}}\right\} .
$$

We define the function $\tilde{R}_{\text {cnt }}: \mathbb{R}_{++} \times \mathbb{R}_{+} \rightarrow \mathbb{R}_{+}$like $\tilde{R}(\cdot, \cdot)$ except that its first argument $\left(D_{\mathrm{d}}\right)$ is strictly positive; the minimum is replaced by an infimum; and the size of the auxiliary alphabet $\mathcal{Z}$ can be unbounded. Thus,

$$
\tilde{R}_{\mathrm{cnt}}\left(D_{\mathrm{d}}, D_{\mathrm{e}}\right) \triangleq \inf _{Z, \phi, \psi} I(X ; Z \mid Y)
$$


where the infimum is over all choices ${ }^{5}$ of the random variable $Z$ and functions $\phi, \psi$ satisfying

$$
\begin{aligned}
& \mathrm{E}\left[\left(X-\hat{X}_{\mathrm{d}}\right)^{2}\right] \leq D_{\mathrm{d}}, \\
& \mathrm{E}\left[\left(\hat{X}_{\mathrm{d}}-\hat{X}_{\mathrm{e}}\right)^{2}\right] \leq D_{\mathrm{e}}, \\
& Z \multimap-X \multimap-Y,
\end{aligned}
$$

where

$$
\begin{aligned}
& \hat{X}_{\mathrm{d}} \triangleq \phi(Y, Z), \\
& \hat{X}_{\mathrm{e}} \triangleq \psi(X, Z) .
\end{aligned}
$$

In analogy to Proposition 5 we have:

Lemma 13: Over $\mathbb{R}_{++} \times \mathbb{R}_{+}$the function $\tilde{R}_{\mathrm{cnt}}\left(D_{\mathrm{d}}, D_{\mathrm{e}}\right)$ is finite, monotonic in each of its arguments, and convex.

Proof: The function is bounded by the rate-distortion function of the Gaussian source without side information. The proof of monotonicity is identical to the proof of monotonicity in Proposition 5. The proof of convexity is also very similar: only a minor change is needed to account for the fact that, prima facie, the infimum need not be achieved.

The following lemma provides an explicit expression for $\tilde{R}_{\text {cnt }}\left(D_{\mathrm{d}}, D_{\mathrm{e}}\right)$ when (111) holds.

Lemma 14: If $D_{\mathrm{d}}>0$ and $D_{\mathrm{e}} \geq 0$ satisfy (111), then

$$
\tilde{R}_{\text {cnt }}\left(D_{\mathrm{d}}, D_{\mathrm{e}}\right)=\frac{1}{2} \log ^{+}\left(\frac{\sigma_{X}^{2}}{\sigma_{X}^{2}+\sigma_{U}^{2}} \frac{\sigma_{U}^{2}+D_{\mathrm{d}}-2 \sqrt{\sigma_{U}^{2} D_{\mathrm{e}}}}{D_{\mathrm{d}}-D_{\mathrm{e}}}\right) .
$$

Proof of Lemma 14: We first prove

$$
\tilde{R}_{\mathrm{cnt}}\left(D_{\mathrm{d}}, D_{\mathrm{e}}\right) \leq \frac{1}{2} \log ^{+}\left(\frac{\sigma_{X}^{2}}{\sigma_{X}^{2}+\sigma_{U}^{2}} \frac{\sigma_{U}^{2}+D_{\mathrm{d}}-2 \sqrt{\sigma_{U}^{2} D_{\mathrm{e}}}}{D_{\mathrm{d}}-D_{\mathrm{e}}}\right) .
$$

To this end, we present a choice for $Z, \hat{X}_{\mathrm{d}}, \hat{X}_{\mathrm{e}}$ that satisfies the constraints (113) and is such that the objective function $I(X ; Z \mid Y)$ in (112) evaluates to the RHS of (115). Our choice depends on whether

$$
D_{\mathrm{d}} \geq \sigma_{X}^{2}\left(1-\sqrt{\frac{D_{\mathrm{e}}}{\sigma_{U}^{2}}}\right)^{2}+D_{\mathrm{e}}
$$

or

$$
D_{\mathrm{d}}<\sigma_{X}^{2}\left(1-\sqrt{\frac{D_{\mathrm{e}}}{\sigma_{U}^{2}}}\right)^{2}+D_{\mathrm{e}} .
$$

In the first case (116) the RHS of (115) evaluates to 0 , whereas in the second case (117) it is positive.

When $D_{\mathrm{d}}$ and $D_{\mathrm{e}}$ satisfy (116), a suitable choice is-as in (83) and (84) in the proof of the direct part-

$$
Z=\emptyset, \quad \hat{X}_{\mathrm{e}}^{n}=\sqrt{\frac{D_{\mathrm{e}}}{\sigma_{U}^{2}}} X^{n}, \quad \hat{X}_{\mathrm{d}}^{n}=\sqrt{\frac{D_{\mathrm{e}}}{\sigma_{U}^{2}}} Y^{n} .
$$

\footnotetext{
${ }^{5}$ To be more precise we should specify the set where $Z$ may take value, and we must restrict the functions $\phi$ and $\psi$ to be measurable. In the converse $Z$ will correspond to the tuple $\left(M, Y^{i-1}, Y_{i+1}^{n}\right)$, and we can therefore restrict $Z$ here to be the space where such tuples take value.
}

When $D_{\mathrm{d}}$ and $D_{\mathrm{e}}$ satisfy (117), a suitable choice is-as in (97) and (106) in the direct part-

$$
Z=a(X+W), \quad \hat{X}_{\mathrm{e}}=b X+Z, \quad \hat{X}_{\mathrm{d}}=b Y+Z,
$$

where $W$ is a centered Gaussian of variance $\sigma_{W}^{2}=$ $\frac{\sigma_{X}^{2}\left(D_{\mathrm{d}}-D_{\mathrm{e}}\right)}{\sigma_{X}^{2}\left(1-\sqrt{D_{\mathrm{e}} / \sigma_{U}^{2}}\right)^{2}+D_{\mathrm{e}}-D_{\mathrm{d}}}$ and independent of the pair $(X, Y)$ and where $b=\sqrt{D_{\mathrm{e}} / \sigma_{U}^{2}}$ and $a=\frac{\sigma_{X}^{2}}{\sigma_{X}^{2}+\sigma_{W}^{2}}(1-b)$. That this choice has the desired properties follows by (102)-(104) and (107).

Having established (115), we now complete the proof of the lemma by proving the reverse inequality

$$
\tilde{R}_{\text {cnt }}\left(D_{\mathrm{d}}, D_{\mathrm{e}}\right) \geq \frac{1}{2} \log ^{+}\left(\frac{\sigma_{X}^{2}}{\sigma_{X}^{2}+\sigma_{U}^{2}} \frac{\sigma_{U}^{2}+D_{\mathrm{d}}-2 \sqrt{\sigma_{U}^{2} D_{\mathrm{e}}}}{D_{\mathrm{d}}-D_{\mathrm{e}}}\right) .
$$

Since rates are nonnegative, it suffices to prove

$$
\tilde{R}_{\text {cnt }}\left(D_{\mathrm{d}}, D_{\mathrm{e}}\right) \geq \frac{1}{2} \log \left(\frac{\sigma_{X}^{2}}{\sigma_{X}^{2}+\sigma_{U}^{2}} \frac{\sigma_{U}^{2}+D_{\mathrm{d}}-2 \sqrt{\sigma_{U}^{2} D_{\mathrm{e}}}}{D_{\mathrm{d}}-D_{\mathrm{e}}}\right)
$$

where $\log ^{+}$has been replaced by $\log$.

Since the joint law of $(X, Y)$ is fixed and is a bivariate Gaussian law

$$
\begin{aligned}
I(X ; Z \mid Y) & =h(X \mid Y)-h(X \mid Y, Z) \\
& =\frac{1}{2} \log \left(2 \pi e \frac{\sigma_{X}^{2} \sigma_{U}^{2}}{\sigma_{X}^{2}+\sigma_{U}^{2}}\right)-h(X \mid Y, Z) .
\end{aligned}
$$

Consequently, (121) is equivalent to

$$
\Omega \leq \frac{1}{2} \log \left(2 \pi e \sigma_{U}^{2} \frac{D_{\mathrm{d}}-D_{\mathrm{e}}}{\sigma_{U}^{2}+D_{\mathrm{d}}-2 \sqrt{\sigma_{U}^{2} D_{\mathrm{e}}}}\right),
$$

where $\Omega$ is defined as

$$
\Omega \triangleq \sup _{Z, \phi, \psi} h(X \mid Y, Z)
$$

under the same constraints (113) that define $\tilde{R}_{\text {cnt }}\left(D_{\mathrm{d}}, D_{\mathrm{e}}\right)$ in (112).

To prove (123) we first note that, since $\hat{X}_{\mathrm{d}}$ is a deterministic function of $(Y, Z)$,

$$
\begin{aligned}
h(X \mid Y, Z) & =h\left(X-\hat{X}_{\mathrm{d}} \mid Y, Z, \hat{X}_{\mathrm{d}}\right) \\
& =h\left(X-\hat{X}_{\mathrm{d}} \mid X-\hat{X}_{\mathrm{d}}+U, Z, \hat{X}_{\mathrm{d}}\right) \\
& \leq h\left(X-\hat{X}_{\mathrm{d}} \mid X-\hat{X}_{\mathrm{d}}+U\right)
\end{aligned}
$$

where in the second line we recalled that $Y=X+U$ (69), and where the last line follows because conditioning cannot increase differential entropy.

The Markov condition $Z-\circ-X-\circ-Y$ (113c) and the fact that $Y=X+U(69)$ imply that

$$
Z \multimap-O-U \text {. }
$$

This, combined with the assumption that $U$ is independent of $X$, implies that $U$ is independent of $(X, Z)$. And since $\hat{X}_{\mathrm{e}}$ is a function of $(X, Z)$,

$$
U \text { and }\left(\hat{X}_{\mathrm{e}}, X, Z\right) \text { are independent. }
$$


This independence implies that $U$ is independent of $\left(X-\hat{X}_{\mathrm{e}}\right)$. This latter independence and the fact that $X-\hat{X}_{\mathrm{d}}$ can be expressed as $-\left(\hat{X}_{\mathrm{d}}-\hat{X}_{\mathrm{e}}-\left(X-\hat{X}_{\mathrm{e}}\right)\right)$ implies that

$$
\operatorname{Cov}\left(X-\hat{X}_{\mathrm{d}}, U\right)=-\operatorname{Cov}\left(\hat{X}_{\mathrm{d}}-\hat{X}_{\mathrm{e}}, U\right) \text {. }
$$

From (130), (113b), the fact that the variance of a random variable cannot exceed its second moment, and the fact that the magnitude of a correlation coefficient cannot exceed 1 , it follows that

$$
\left|\operatorname{Cov}\left(X-\hat{X}_{\mathrm{d}}, U\right)\right|^{2} \leq D_{\mathrm{e}} \sigma_{U}^{2} .
$$

From (127) and (131) we thus obtain

$$
\Omega \leq \Gamma
$$

where $\Gamma$ is defined as

$$
\Gamma \triangleq \sup _{\hat{X}_{\mathrm{d}}} h\left(X-\hat{X}_{\mathrm{d}} \mid X-\hat{X}_{\mathrm{d}}+U\right)
$$

subject to the relaxed constraints

$$
\begin{aligned}
\operatorname{Var}\left(X-\hat{X}_{\mathrm{d}}\right) & \leq D_{\mathrm{d}}, \\
\left|\operatorname{Cov}\left(X-\hat{X}_{\mathrm{d}}, U\right)\right|^{2} & \leq D_{\mathrm{e}} \sigma_{U}^{2} .
\end{aligned}
$$

We now proceed to study $\Gamma$. Define

$$
A \triangleq X-\hat{X}_{\mathrm{d}}
$$

so

$$
\Gamma=\sup _{A} h(A \mid A+U)
$$

subject to

$$
\begin{aligned}
\operatorname{Var}(A) & \leq D_{\mathrm{d}}, \\
|\operatorname{Cov}(A, U)|^{2} & \leq D_{\mathrm{e}} \sigma_{U}^{2} .
\end{aligned}
$$

By the conditional max-entropy theorem [16], the supremum in (136) is achieved when $(A, U)$ are jointly Gaussian, as we henceforth assume. As we next argue, the lemma's hypothesis that (111) holds implies that the choice of $A$ as $-U$ is not in the feasible set. Indeed, with this choice $|\operatorname{Cov}(A, U)|^{2}$ is equal to $\sigma_{U}^{4}$, which violates (137b) because (111) and (98) imply

$$
D_{\mathrm{e}}<\min \left\{\sigma_{U}^{2}, D_{\mathrm{d}}\right\} .
$$

We thus assume in the following that $A$ is jointly Gaussian with $U$ and that $A \neq-U$. Consequently,

$$
\begin{aligned}
& h(A \mid A+U) \\
& =\frac{1}{2} \log \left(2 \pi e\left(\sigma_{U}^{2}-\frac{\left(\sigma_{U}^{2}+\kappa_{A U}\right)^{2}}{\sigma_{A}^{2}+\sigma_{U}^{2}+2 \kappa_{A U}}\right)\right) \\
& =\frac{1}{2} \log \left(2 \pi e \frac{\sigma_{A}^{2} \sigma_{U}^{2}-\kappa_{A U}^{2}}{\sigma_{A}^{2}+\sigma_{U}^{2}+2 \kappa_{A U}}\right)
\end{aligned}
$$

where $\sigma_{A}^{2} \triangleq \operatorname{Var}(A)$ and $\kappa_{A U} \triangleq \operatorname{Cov}(A, U)$.

We can thus rewrite the optimization problem in (133) as

$$
\Gamma=\sup _{\kappa_{A U}, \sigma_{A}^{2}} \frac{1}{2} \log \left(2 \pi e \frac{\sigma_{A}^{2} \sigma_{U}^{2}-\kappa_{A U}^{2}}{\sigma_{A}^{2}+\sigma_{U}^{2}+2 \kappa_{A U}}\right)
$$

subject to

$$
\begin{aligned}
& 0 \leq \sigma_{A}^{2} \leq D_{\mathrm{d}}, \\
& 0 \leq\left|\kappa_{A U}\right|^{2} \leq D_{\mathrm{e}} \sigma_{U}^{2}, \\
& 0 \leq\left|\kappa_{A U}\right|^{2} \leq \sigma_{A}^{2} \sigma_{U}^{2} .
\end{aligned}
$$

(We have to add the last constraint because the magnitude of a correlation coefficient cannot exceed one.) For fixed $\kappa_{A U}$, the objective function in (141) is monotonically increasing in $\sigma_{A}^{2}$ (see also (139)), and so is the RHS of Constraint (144). Therefore, it is optimal to choose in (141)

$$
\sigma_{A}^{2}=D_{\mathrm{d}} .
$$

Substituting this choice in (141) and (144) yields

$$
\Gamma=\sup _{\kappa_{A U}} \frac{1}{2} \log \left(2 \pi e \frac{D_{\mathrm{d}} \sigma_{U}^{2}-\kappa_{A U}^{2}}{D_{\mathrm{d}}+\sigma_{U}^{2}+2 \kappa_{A U}}\right)
$$

subject to (143) and

$$
0 \leq\left|\kappa_{A U}\right|^{2} \leq D_{\mathrm{d}} \sigma_{U}^{2} .
$$

Notice that, whenever (111) holds, the RHS of (143) is upperbounded by the square of $\min \left\{D_{\mathrm{d}}, \sigma_{U}^{2}\right\}$. Consequently,

$$
((111) \text { and }(143)) \Rightarrow\left(\left|\kappa_{A U}\right|<\min \left\{D_{\mathrm{d}}, \sigma_{U}^{2}\right\}\right) .
$$

Since the RHS of (148) implies (147),

$$
((111) \text { and }(143)) \Rightarrow(147),
$$

and Constraint (147) is redundant. We therefore ignore Constraint (147) and study the maximization in (146) subject to (143) only.

To this end, we compute the derivative of the objective function in (146) with respect to $\kappa_{A U}$ :

$$
\begin{gathered}
\frac{\mathrm{d}}{\mathrm{d} \kappa_{A U}}\left(\frac{1}{2} \log \left(2 \pi e \frac{D_{\mathrm{d}} \sigma_{U}^{2}-\kappa_{A U}^{2}}{D_{\mathrm{d}}+\sigma_{U}^{2}+2 \kappa_{A U}}\right)\right) \\
=\frac{-\left(D_{\mathrm{d}}+\kappa_{A U}\right)\left(\sigma_{U}^{2}+\kappa_{A U}\right)}{\left(D_{\mathrm{d}}+\sigma_{U}^{2}+2 \kappa_{A U}\right)\left(D_{\mathrm{d}} \sigma_{U}^{2}-\kappa_{A U}^{2}\right)} .
\end{gathered}
$$

By (148), the derivative in (150) is negative for all feasible $\kappa_{A U}$. Hence, the objective function in (146) is decreasing on the (symmetric) interval of interest (143), and it is optimal to choose

$$
\kappa_{A U}=-\sqrt{D_{\mathrm{e}} \sigma_{U}^{2}} .
$$

The optimality of this choice allows us to evaluate $\Gamma$ via (146) and hence to upper-bound $\Omega$ via (132). This yields the desired bound (123), which establishes the lemma.

Proof of Converse When (111) Holds: Using Lemma 13 and Lemma 14 we can follow the steps of the proof in Section II-D of the converse part of Theorem 6. The remaining technicality is continuity. Continuity in the interior, i.e., on $\mathbb{R}_{++} \times \mathbb{R}_{++}$follows from convexity. It thus only remains to establish continuity when $D_{\mathrm{d}}>0$, (111) holds, and $D_{\mathrm{e}}$ is zero. This can be done by inspecting (114). 


\section{More And More-General Constraints}

So far we have only studied settings with two distortion measures, one of which - the decoder-side distortion measure $d_{\mathrm{d}}\left(x, \hat{x}_{\mathrm{d}}\right)$-depends on the source symbol and the decoder's reconstruction, and the other-the encoder-side distortion measure $d_{\mathrm{e}}\left(\hat{x}_{\mathrm{d}}, \hat{x}_{\mathrm{e}}\right)$-depends on the decoder's and the encoder's reconstruction symbols. In this section we extend our setting to allow for more than two distortion measures and to allow for distortions that depend on all three symbols: the source symbol $x$, the decoder's reconstruction symbol $\hat{x}_{\mathrm{d}}$, and the encoder's reconstruction symbol $\hat{x}_{\mathrm{e}}$. We shall also allow the reconstruction alphabets to differ. But all alphabets are assumed finite.

\section{A. Problem Statement}

The new setup differs from the setup in Section II in two ways.

- The encoder-side reconstruction $\hat{X}_{\mathrm{e}}^{n}$ and the decoderside reconstruction $\hat{X}_{\mathrm{d}}^{n}$ take value in the finite alphabets $\hat{\mathcal{X}}_{\mathrm{e}}^{n}$ and $\hat{\mathcal{X}}_{\mathrm{d}}^{n}$ which can be different.

- There are $K$ (possibly larger than 2) distortion constraints specified by the $K$ distortion measures

$$
d_{k}: \mathcal{X} \times \mathcal{X}_{\mathrm{d}} \times \mathcal{X}_{\mathrm{e}} \rightarrow \mathbb{R}_{+}, \quad k \in\{1, \ldots, K\}
$$

and the corresponding $K$ maximal-allowed distortions $D_{1}, \ldots, D_{K}$ (all of which are assumed to be nonnegative).

We say that the tuple $\left(R, D_{1}, \ldots, D_{K}\right)$ is achievable if for every $\epsilon>0$ and sufficiently large $n$ there exist a message set $\mathcal{M}$ of size $|\mathcal{M}| \leq 2^{n(R+\epsilon)}$ and functions

$$
\begin{aligned}
& f^{(n)}: \mathcal{X}^{n} \rightarrow \mathcal{M} \\
& \phi^{(n)}: \mathcal{M} \times \mathcal{Y}^{n} \rightarrow \hat{\mathcal{X}}_{\mathrm{d}}^{n} \\
& \psi^{(n)}: \mathcal{X}^{n} \rightarrow \hat{\mathcal{X}}_{\mathrm{e}}^{n}
\end{aligned}
$$

such that the message $M=f^{(n)}\left(X^{n}\right)$ and the reconstruction sequences $\hat{X}_{\mathrm{d}}^{n}=\phi^{(n)}\left(M, Y^{n}\right)$ and $\hat{X}_{\mathrm{e}}^{n}=\psi^{(n)}\left(X^{n}\right)$ satisfy:

$$
\frac{1}{n} \sum_{i=1}^{n} \mathrm{E}\left[d_{k}\left(X_{i}, \hat{X}_{\mathrm{d}, i}, \hat{X}_{\mathrm{e}, i}\right)\right] \leq D_{k}+\epsilon, \quad k \in\{1, \ldots, K\} .
$$

In analogy to Assumption 1, we shall assume:

Assumption 2: To each $x \in \mathcal{X}$ corresponds some $\hat{x}_{\mathrm{d}} \in \hat{X}_{\mathrm{d}}$ and some $\hat{x}_{\mathrm{e}} \in \hat{X}_{\mathrm{e}}$ satisfying

$$
d_{k}\left(x, \hat{x}_{\mathrm{d}}, \hat{x}_{\mathrm{e}}\right)=0, \quad k \in\{1, \ldots, K\} .
$$

We seek the smallest rate $R$ for which the tuple $\left(R, D_{1}, \ldots, D_{K}\right)$ is achievable. This is defined as follows. Given a maximal-allowed-distortion tuple $\left(D_{1}, \ldots, D_{K}\right)$, let

$$
\begin{aligned}
& \mathcal{R}_{\text {Ext }}\left(D_{1}, \ldots, D_{K}\right) \\
& \quad \triangleq\left\{R \in \mathbb{R}_{+}:\left(R, D_{1}, \ldots, D_{K}\right) \text { is achievable }\right\} .
\end{aligned}
$$

Assumption 2 implies that the set $\mathcal{R}_{\text {Ext }}\left(D_{1}, \ldots, D_{K}\right)$ contains all rates exceeding $H(X \mid Y)$ and is thus nonempty. The ratedistortions function $R_{\text {Ext }}$ can now be defined as

$$
R_{\mathrm{Ext}}\left(D_{1}, \ldots, D_{K}\right) \triangleq \min _{R \in \mathcal{R}_{\mathrm{Ext}}\left(D_{1}, \ldots, D_{K}\right)} R,
$$

where the minimum exists because the region $\mathcal{R}_{\text {Ext }}\left(D_{1}, \ldots, D_{K}\right) \subset \mathbb{R}_{+}$is nonempty, closed, and bounded from below by 0 .

\section{B. Result}

To describe the rate-distortions function for the extended setup of Section IV-A, we next introduce the function $\tilde{R}_{\text {Ext }}\left(D_{1}, \ldots, D_{K}\right)$.

Given the joint law $P_{X Y}$ of the source and side information, and given the distortion measures $d_{1}, \ldots, d_{K}$, this function is defined as

$$
\tilde{R}_{\text {Ext }}\left(D_{1}, \ldots, D_{K}\right)=\min _{U, Z, \phi, \psi}(I(X ; Z)-I(Y ; Z))
$$

where the minimization is over all discrete auxiliary random variables $Z$ and $U$ satisfying

$$
(U, Z) \multimap-X \multimap Y
$$

and over all functions $\phi: \mathcal{Y} \times \mathcal{Z} \rightarrow \hat{\mathcal{X}}_{\mathrm{d}}$ and $\psi: \mathcal{X} \times \mathcal{Z} \times \mathcal{U} \rightarrow \hat{\mathcal{X}}_{\mathrm{e}}$ that simultaneously satisfy the $K$ distortion constraints

$$
\mathrm{E}\left[d_{k}(X, \phi(Y, Z), \psi(X, Z, U))\right] \leq D_{k}, \quad k \in\{1, \ldots, K\} .
$$

The following proposition provides cardinality bounds on the support sets of the auxiliary random variables.

Proposition 15 (Cardinality Bounds): The minimum defining $\tilde{R}_{\text {Ext }}\left(D_{1}, \ldots, D_{K}\right)$ is not increased if we restrict the cardinality of the support set $\mathcal{Z}$ of $Z$ to

$$
|\mathcal{Z}| \leq|\mathcal{X}||\mathcal{U}|+K+1
$$

and the cardinality of the support set $\mathcal{U}$ of $U$ to

$$
|\mathcal{U}| \leq K .
$$

Proof: The cardinality bound on $\mathcal{Z}$ can be justified using the convex cover method [9]. The cardinality bound on $\mathcal{U}$ is proved in Appendix D.

Remark 16 (Improved Cardinality Bound): The cardinality bound on $\mathcal{U}$ can be strengthened: $|\mathcal{U}|$ need not exceed the number of distortion constraints in (154) that depend on $\hat{X}_{\mathrm{e}, i}$. The latter number equals 1 in the original setup of Section II thus allowing us to recover Theorem 6.

Proposition 17 (Key Properties of the Function $\tilde{R}_{\text {Ext }}$ ): The function $\tilde{R}_{\text {Ext }}: \mathbb{R}_{+}^{K} \rightarrow \mathbb{R}_{+}$is bounded from above by $H(X \mid Y)$; it is nondecreasing in the distortions

$$
\begin{aligned}
& \left(D_{1}^{\prime} \geq D_{1}, \ldots, D_{K}^{\prime} \geq D_{K}\right) \\
& \quad \Longrightarrow\left(\tilde{R}_{\text {Ext }}\left(D_{1}^{\prime}, \ldots, D_{K}^{\prime}\right) \leq \tilde{R}_{\mathrm{Ext}}\left(D_{1}, \ldots, D_{K}\right)\right) ;
\end{aligned}
$$

and it is convex and continuous.

Proof: The proof is similar to the proof of Proposition 5 in Appendix B and is omitted.

Theorem 18: The rate-distortions function for the setup in Section IV-A is equal to $\tilde{R}_{\text {Ext }}\left(D_{1}, \ldots, D_{K}\right)$ :

$$
R_{\text {Ext }}\left(D_{1}, \ldots, D_{K}\right)=\tilde{R}_{\text {Ext }}\left(D_{1}, \ldots, D_{K}\right) .
$$

Compared to the rate-distortions function of our original problem $\tilde{R}\left(D_{\mathrm{d}}, D_{\mathrm{e}}\right)$ in (25), the definition of the rate-distortions 
function $\tilde{R}_{\text {Ext }}\left(D_{1}, \ldots, D_{K}\right)$ in (158) involves an extra auxiliary random variable $U$. This auxiliary $U$ shows up as an additional argument in the encoder's reconstruction function $\psi$ and is subject to the Markov chain (159). ${ }^{6}$ Intuitively, the auxiliary $U$ is needed because there might not be a deterministic encoder-side reconstruction function that matches all the $K$ distortion constraints simultaneously, but instead there are several (in fact $K$ ) different reconstruction functions that meet the $K$ distortion constraints only on average.

Our results extend also to vector-valued distortion measures as considered in [18].

Proof of Theorem 18: The achievability, i.e., that

$$
R_{\text {Ext }}\left(D_{1}, \ldots, D_{K}\right) \leq \tilde{R}_{\text {Ext }}\left(D_{1}, \ldots, D_{K}\right),
$$

can be proved using a scheme that is similar to the one that was sketched in the proof of Theorem 6. The only difference is that, to produce the reconstruction sequence $\hat{X}_{\mathrm{e}}^{n}$, the encoder applies the function $\psi$ component-wise to the tuple $\left(X^{n}, Z^{* n}, U^{n}\right)$, where, conditional on $\left(X^{n}, Z^{* n}\right)$, the components of the sequence $U^{n}$ are generated independently according to the conditional law $P_{U \mid Z, X}$. The analysis of this scheme is omitted.

We next prove the converse, i.e., that

$$
R_{\text {Ext }}\left(D_{1}, \ldots, D_{K}\right) \geq \tilde{R}_{\text {Ext }}\left(D_{1}, \ldots, D_{K}\right) .
$$

Fix some positive $\epsilon$, a blocklength $n$, and a rate $R$. Let $\mathcal{M}$ be a message set of size $|\mathcal{M}| \leq 2^{n(R+\epsilon)}$, and let $f^{(n)}, \phi^{(n)}$, and $\psi^{(n)}$ be encoding and reconstruction functions as in (153) that satisfy the $K$ distortion constraints in (154). For every $i \in\{1, \ldots, n\}$, define $Z_{i}$ in (44)

$$
Z_{i} \triangleq\left(M, Y^{i-1}, Y_{i+1}^{n}\right)
$$

and define $U_{i}$ as $X_{\backslash i}$, i.e.,

$$
U_{i} \triangleq\left(X_{1}^{i-1}, X_{i+1}^{n}\right)
$$

Notice that for every $i \in\{1, \ldots, n\}$

$$
\left(U_{i}, Z_{i}\right) \multimap-X_{i} \multimap-Y_{i} .
$$

Also, following the steps in (34)-(43), we can conclude that

$$
n(R+\epsilon) \geq \sum_{i=1}^{n} I\left(X_{i} ; Z_{i}\right)-I\left(Y_{i} ; Z_{i}\right) .
$$

We further define-as in Section II-D— $\phi_{i}^{(n)}$ to be the function that maps $\left(M, Y^{n}\right)$ to the $i$-th symbol of $\phi^{(n)}\left(M, Y^{n}\right)$ and $\psi_{i}^{(n)}$ to be the function that maps $X^{n}$ to the $i$-th symbol of $\psi^{(n)}\left(X^{n}\right)$. Then, the symbol $\phi_{i}^{(n)}\left(M, Y^{n}\right)$ can be written as

$$
\phi_{i}\left(Y_{i}, Z_{i}\right) \triangleq \phi_{i}^{(n)}\left(M, Y^{n}\right)
$$

and $\psi_{i}^{(n)}\left(X^{n}\right)$ can be written as

$$
\psi_{i}\left(X_{i}, Z_{i}, U_{i}\right) \triangleq \psi_{i}^{(n)}\left(X^{n}\right),
$$

\footnotetext{
${ }^{6}$ The function $\tilde{R}_{\text {Ext }}\left(D_{1}, \ldots, D_{K}\right)$ could also be defined without the auxiliary $U$, if instead $\psi$ was allowed to be a randomized function of $X$ and $Z$.
}

for some functions $\phi_{i}$ and $\psi_{i}$ with arguments in the respective domains. We finally define for each $k \in\{1, \ldots, K\}$ and $i \in$ $\{1, \ldots, n\}$

$$
D_{k, i} \triangleq \mathrm{E}\left[d_{k}\left(X_{i}, \phi_{i}^{(n)}\left(M, Y^{n}\right), \psi_{i}^{(n)}\left(X^{n}\right)\right)\right],
$$

where $\mathrm{E}[\cdot]$ is with respect to $P_{X^{n}} Y^{n}$. Notice that

$$
\sum_{i=1}^{n} D_{k, i} \leq D_{k}+\epsilon, \quad k \in\{1, \ldots, K\}
$$

because the chosen encoding and reconstruction functions $f^{(n)}, \phi^{(n)}$, and $\psi^{(n)}$ satisfy (154). Moreover, by definitions (170)-(172),

$$
\mathrm{E}\left[d_{k}\left(X_{i}, \phi_{i}\left(Y_{i}, Z_{i}\right), \psi_{i}\left(X_{i}, Z_{i}, U_{i}\right)\right)\right]=D_{k, i},
$$

where E[·] is with respect to $P_{X_{i} Y_{i}} P_{U_{i} Z_{i} \mid X_{i}}$.

Combining (169) and (174) with the definition of $\tilde{R}_{\text {Ext }}$, we obtain

$$
\begin{aligned}
n(R+\epsilon) & \geq \sum_{i=1}^{n} I\left(X_{i} ; Z_{i}\right)-I\left(Y_{i} ; Z_{i}\right) \\
& \geq \sum_{i=1}^{n} \tilde{R}_{\mathrm{Ext}}\left(D_{1, i}, \ldots, D_{K, i}\right) \\
& \geq n \tilde{R}_{\mathrm{Ext}}\left(\frac{1}{n} \sum_{i=1}^{n} D_{1, i}, \ldots, \frac{1}{n} \sum_{i=1}^{n} D_{K, i}\right) \\
& \geq n \tilde{R}_{\mathrm{Ext}}\left(D_{1}+\epsilon, \ldots, D_{K}+\epsilon\right),
\end{aligned}
$$

where the last two inequalities follow by the convexity and the monotonicity of $\tilde{R}_{\text {Ext }}$ and by (173). By the continuity of $\tilde{R}_{\text {Ext }}$ and because $\epsilon>0$ and the blocklength $n$ are arbitrary, the converse (165) follows immediately from (178).

\section{APPENDIX A}

\section{Proof of COROLlary 7}

When $d_{\mathrm{e}}(\cdot, \cdot)$ is the Hamming distortion and $D_{\mathrm{e}}=0$, our average-per-symbol distortion constraint (12) is less stringent than the block-distortion constraint (24) in Steinberg's setup (Remark 3). Consequently,

$$
R_{\text {cr }}\left(D_{\mathrm{d}}\right) \geq R\left(D_{\mathrm{d}}, 0\right) .
$$

It remains to prove the reverse inequality. Let $Z, \phi$, and $\psi$ be minimizers of $R\left(D_{\mathrm{d}}, 0\right)$, so

$$
\begin{gathered}
R\left(D_{\mathrm{d}}, 0\right)=I(X ; Z)-I(Y ; Z) \\
\mathrm{E}\left[d_{\mathrm{d}}(X, \phi(Y, Z))\right] \leq D_{\mathrm{d}} \\
\phi(Y, Z)=\psi(X, Z) \quad \text { w.p. } 1 \\
Z \multimap-\triangle \multimap-Y .
\end{gathered}
$$

To prove the reverse inequality we shall upper-bound $R_{\mathrm{cr}}\left(D_{\mathrm{d}}\right)$ by showing that

$$
\hat{X} \triangleq \phi(Y, Z)
$$

is feasible in the minimization (21) that defines it.

From the definition of $\hat{X}$ (181) and from (180c), it follows that $\hat{X}$ is computable (w.p. 1) from $(X, Z)$. This combines with (180d) to establish that

$$
(\hat{X}, Z) \multimap-\circ \multimap Y
$$


and, a fortiori, that

$$
\hat{X} \multimap \circ-\circ-Y \text {. }
$$

And by (180b) and (181),

$$
\mathrm{E}\left[d_{\mathrm{d}}(X, \hat{X})\right] \leq D_{\mathrm{d}}
$$

It follows from (183) that $\hat{X}$ is feasible in the minimization (21) defining $R_{\mathrm{cr}}\left(D_{\mathrm{d}}\right)$ and thus

$$
\begin{aligned}
R_{\mathrm{cr}}\left(D_{\mathrm{d}}\right) & \leq I(X ; \hat{X})-I(Y ; \hat{X}) \\
& =I(X ; \hat{X} \mid Y) \\
& \leq I(X ; Z \mid Y) \\
& =I(X ; Y)-I(X ; Z) \\
& =R\left(D_{\mathrm{d}}, 0\right)
\end{aligned}
$$

where (185) follows from (183a); where (186) follows, by the (conditional) data processing inequality, from

$$
\hat{X} \multimap(Y, Z) \multimap-X
$$

(which holds by (181)); where (187) follows from (180d); and (188) follows from (180a). Inequalities (179) and (188) establish the corollary.

\section{APPENDIX B}

\section{PROOF OF PROPOSITION 5}

That $\tilde{R}\left(D_{\mathrm{d}}, D_{\mathrm{e}}\right)$ is bounded by $H(X \mid Y)$ is just a restatement of (29). Monotonicity holds because the feasible set in the minimization defining $\tilde{R}\left(D_{\mathrm{d}}, D_{\mathrm{e}}\right)$ is enlarged (or is unaltered) when $D_{\mathrm{d}}$ and/or $D_{\mathrm{e}}$ are increased.

As to the convexity, let $Z^{(1)}, \phi^{(1)}, \psi^{(1)}$ and $Z^{(2)}, \phi^{(2)}, \psi^{(2)}$ be the random variables and functions that achieve the minima in the definitions of $\tilde{R}\left(D_{\mathrm{d}}^{(1)}, D_{\mathrm{e}}^{(1)}\right)$ and $\tilde{R}\left(D_{\mathrm{d}}^{(2)}, D_{\mathrm{e}}^{(2)}\right)$. Let $Q \sim \operatorname{Bernoulli}(\lambda)$ be independent of $\left(X, Y, Z^{(1)}, Z^{(2)}\right)$. Define

$$
Z \triangleq\left(Q, Z^{(Q)}\right)
$$

and the functions

$$
\begin{gathered}
\phi(Y, Z) \triangleq \phi^{(Q)}\left(Y, Z^{(Q)}\right) \\
\psi(X, Z) \triangleq \psi^{(Q)}\left(X, Z^{(Q)}\right) .
\end{gathered}
$$

Then

$$
\begin{aligned}
& Z \multimap X \multimap Y ; \\
& \begin{aligned}
E\left[d_{\mathrm{d}}(X, \phi\right. & (Y, Z))] \\
= & \lambda E\left[d_{\mathrm{d}}\left(X, \phi^{(1)}\left(Y, Z^{(1)}\right)\right)\right] \\
& +(1-\lambda) E\left[d_{\mathrm{d}}\left(X, \phi^{(2)}\left(Y, Z^{(2)}\right)\right)\right] \\
\leq & \lambda D_{\mathrm{d}}^{(1)}+(1-\lambda) D_{\mathrm{d}}^{(2)} ;
\end{aligned}
\end{aligned}
$$

and

$$
\begin{aligned}
E[ & \left.d_{\mathrm{e}}(\phi(Y, Z), \psi(X, Z))\right] \\
= & \lambda E\left[d_{\mathrm{e}}\left(\phi^{(1)}\left(Y, Z^{(1)}\right), \psi^{(1)}\left(X, Z^{(1)}\right)\right)\right] \\
& +(1-\lambda) E\left[d_{\mathrm{e}}\left(\phi^{(2)}\left(Y, Z^{(2)}\right), \psi^{(2)}\left(X, Z^{(2)}\right)\right)\right] \\
\leq & \lambda D_{\mathrm{e}}^{(1)}+(1-\lambda) D_{\mathrm{e}}^{(2)}
\end{aligned}
$$

so $Z, \phi, \psi$ are feasible for the distortions

$$
\left(\lambda D_{\mathrm{d}}^{(1)}+(1-\lambda) D_{\mathrm{d}}^{(2)}, \lambda D_{\mathrm{e}}^{(1)}+(1-\lambda) D_{\mathrm{e}}^{(2)}\right) .
$$

Consequently,

$$
\begin{aligned}
& \tilde{R}\left(\lambda D_{\mathrm{d}}^{(1)}+(1-\lambda) D_{\mathrm{d}}^{(2)}, \lambda D_{\mathrm{e}}^{(1)}+(1-\lambda) D_{\mathrm{e}}^{(2)}\right) \\
& \leq I(X ; Z)-I(Y ; Z) \\
&= H(X)-H(X \mid Z)-H(Y)+H(Y \mid Z) \\
&= H(X)-H\left(X \mid Z^{(Q)}, Q\right)-H(Y)+H\left(Y \mid Z^{(Q)}, Q\right) \\
&= H(X)-\lambda H\left(X \mid Z^{(1)}\right)-(1-\lambda) H\left(X \mid Z^{(2)}\right) \\
&-H(Y)+\lambda H\left(Y \mid Z^{(1)}\right)+(1-\lambda) H\left(Y \mid Z^{(2)}\right) \\
&= \lambda\left(I\left(X ; Z^{(1)}\right)-I\left(Y ; Z^{(1)}\right)\right) \\
&+(1-\lambda)\left(I\left(X ; Z^{(2)}\right)-I\left(Y ; Z^{(2)}\right)\right) . \\
&= \lambda \tilde{R}\left(D_{\mathrm{d}}^{(1)}, D_{\mathrm{e}}^{(1)}\right)+(1-\lambda) \tilde{R}\left(D_{\mathrm{d}}^{(2)}, D_{\mathrm{e}}^{(2)}\right) .
\end{aligned}
$$

To conclude the proof it remains to prove that $\tilde{R}\left(D_{\mathrm{d}}, D_{\mathrm{e}}\right)$ is continuous on $\mathbb{R}_{+}^{2}$. (Continuity on $\mathbb{R}_{++}^{2}$ is a consequence of the convexity, but we also claim continuity in the closed set $\mathbb{R}_{+}^{2}$.) Since $\mathbb{R}_{+}^{2}$ is locally simplicial (as can be verified by the definition in [17, Sec. 10, p. 84] or using $\left[17\right.$, Th. 20.5 , p. 184]), the convexity of $\tilde{R}\left(D_{\mathrm{d}}, D_{\mathrm{e}}\right)$ on $\mathbb{R}_{+}^{2}$ implies its upper-semicontinuity relative to $\mathbb{R}_{+}^{2}$. It thus remains to prove lower-semicontinuity relative to $\mathbb{R}_{+}^{2}$. That is, we need to show that

$$
\left(D_{\mathrm{d}}^{(\kappa)}, D_{\mathrm{e}}^{(\kappa)}\right) \rightarrow\left(D_{\mathrm{d}}, D_{\mathrm{e}}\right) \quad \text { as } \kappa \rightarrow \infty
$$

implies that there is a subsequence $\left\{\kappa_{\nu}\right\}$ such that

$$
\tilde{R}\left(D_{d}, D_{\mathrm{e}}\right) \leq \lim _{\nu \rightarrow \infty} \tilde{R}\left(D_{\mathrm{d}}^{\left(\kappa_{\nu}\right)}, D_{\mathrm{e}}^{\left(\kappa_{\nu}\right)}\right) .
$$

Let $\phi^{(\kappa)}, \psi^{(\kappa)}, P_{Z \mid X}^{(\kappa)}$ achieve $\tilde{R}\left(D_{\mathrm{d}}^{(\kappa)}, D_{\mathrm{e}}^{(\kappa)}\right)$ with $\mathcal{Z}=$ $\{1, \ldots,|\mathcal{X}|+3\}$. Since there are only a finite number of functions from $\mathcal{Y} \times \mathcal{Z}$ to $\hat{\mathcal{X}}$ and only a finite number of functions from $\mathcal{X} \times \mathcal{Z}$ to $\hat{\mathcal{X}}$, we can choose a subsequence $\left\{\kappa_{\nu}\right\}$ along which: the mappings $\phi^{\left(\kappa_{\nu}\right)}$ do not depend on $v$ and can be thus denoted $\phi$; the mappings $\psi^{\left(\kappa_{\nu}\right)}$ do not depend on $v$ and can be thus denoted $\psi$; and the conditional laws $P_{Z \mid X}^{\left(\kappa_{\nu}\right)}$ converge to some conditional law that we denote $P_{Z \mid X}^{(0)}$. By the continuity of mutual information, $\tilde{R}\left(D_{\mathrm{d}}^{\left(\kappa_{\nu}\right)}, D_{\mathrm{e}}^{\left(\kappa_{\nu}\right)}\right)$ converges to $I(X ; Z)-I(Y ; Z)$ evaluated with respect to $P_{Z \mid X}^{(0)} P_{X Y}$, and $\tilde{R}\left(D_{d}, D_{\mathrm{e}}\right)$ cannot exceed this value because $P_{Z \mid X}^{(0)}, \psi$, and $\phi$ are in the feasible set defining it.

\section{APPENDIX C \\ PROOF OF PROPOSITION 11}

We present and analyze a scheme that achieves the rate-distortions tuples in Proposition 11. Before describing the scheme, we introduce some notation and lemmas on $n$-dimensional spheres.

\section{A. On n-Dimensional Spheres}

An $n$-sphere of radius $r>0$ centered at $\xi \in \mathbb{R}^{n}$ is the set of all vectors $\mathbf{x} \in \mathbb{R}^{n}$ satisfying

$$
\|\mathbf{x}-\boldsymbol{\xi}\|=r .
$$

When the center of the sphere $\boldsymbol{\xi}$ is the origin $\mathbf{0}$, we call it a centered sphere, and when the radius of the sphere is 1 , we call it a unit sphere. 
We denote the angle between two nonzero vectors $\mathbf{u}, \mathbf{v} \in \mathbb{R}^{n}$ by $\varangle(\mathbf{u}, \mathbf{v})$. Its cosine is

$$
\cos \varangle(\mathbf{u}, \mathbf{v}) \triangleq \frac{\langle\mathbf{u}, \mathbf{v}\rangle}{\|\mathbf{u}\|\|\mathbf{v}\|} .
$$

Given a nonzero vector $\mu$ on an $n$-sphere $\mathcal{S}$, the spherical cap of half-angle $\theta$ centered at $\boldsymbol{\mu}$ is the set of all vectors $\mathbf{x}$ on $\mathcal{S}$ satisfying

$$
\varangle(\boldsymbol{\mu}, \mathbf{x}) \leq \theta \text {. }
$$

The surface area of such a spherical cap does not depend on the vector $\boldsymbol{\mu}$ but only on the dimension $n$, the radius of the sphere $r$, and the angle $\theta$. If the radius $r=1$, we denote this surface area by $C_{n}(\theta)$.

We say that a random $n$-vector is uniformly distributed over an $n$-sphere, if it is drawn according to a uniform probability measure over the surface of this sphere.

The proofs of the following four lemmas are based on results in [19] and omitted.

Lemma 19: Let $\boldsymbol{\Psi}$ be uniformly distributed over the centered unit $n$-sphere, and let $\boldsymbol{\mu}$ be a deterministic unit-length vector in $\mathbb{R}^{n}$. Then,

$\operatorname{Pr}[\langle\boldsymbol{\Psi}, \boldsymbol{\mu}\rangle \geq \tau]=\frac{C_{n}(\arccos (\tau))}{C_{n}(\pi)}, \quad 0 \leq \tau \leq 1$.

Lemma 20: For $0 \leq \tau<1$ :

$$
\lim _{n \rightarrow \infty} \frac{1}{n} \log \left(\frac{C_{n}(\arccos (\tau))}{C_{n}(\pi)}\right)=\frac{1}{2} \log \left(1-\tau^{2}\right) .
$$

Lemma 21: Let $f: \mathbb{R} \rightarrow(0,1]$ be such that the limit

$$
-\eta_{1} \triangleq \lim _{n \rightarrow \infty} \frac{1}{n} \log f(n)
$$

exists and $\eta_{1}>0$. Then,

$$
\lim _{n \rightarrow \infty}(1-f(n))^{2^{n \eta_{2}}}= \begin{cases}1 & \text { if } \eta_{1}>\eta_{2} \\ 0 & \text { if } \eta_{1}<\eta_{2}\end{cases}
$$

Lemma 22: For $\theta \in(0, \pi / 2)$

$$
\lim _{n \rightarrow \infty} \frac{C_{n}(\theta)}{C_{n}(\pi)}=0
$$

whereas for $\theta \in(\pi / 2, \pi)$

\section{B. Scheme}

$$
\lim _{n \rightarrow \infty} \frac{C_{n}(\theta)}{C_{n}(\pi)}=1 .
$$

Our scheme has parameters

$$
a, \delta, \sigma_{W}^{2}>0 \text { and } b \geq 0
$$

that must satisfy Conditions (86a) and (86b), which we repeat for convenience here:

$$
\begin{aligned}
(1-a-b)^{2} \sigma_{X}^{2}+a^{2} \sigma_{W}^{2}+b^{2} \sigma_{U}^{2} & \leq D_{\mathrm{d}} \\
b^{2} \sigma_{U}^{2} & \leq D_{\mathrm{e}} .
\end{aligned}
$$

To describe and analyze the scheme we use vector notation. Let $\mathbf{X}$ denote the $n$-dimensional column-vector that results when the source symbols are stacked on top of each other

$$
\mathbf{X} \triangleq\left(\begin{array}{llll}
X_{1} & X_{2} & \ldots & X_{n}
\end{array}\right)^{\top} .
$$

Likewise define the side-information vector $\mathbf{Y}$ and the reconstruction vectors $\hat{\mathbf{X}}_{\mathrm{d}}$, and $\hat{\mathbf{X}}_{\mathrm{e}}$.
1) Codebook Generation: Let

$$
\begin{aligned}
\sigma_{Z}^{2} & \triangleq a^{2}\left(\sigma_{W}^{2}+\sigma_{X}^{2}\right), \\
R^{\prime} & \triangleq \frac{1}{2} \log \left(\frac{\sigma_{X}^{2}+\sigma_{W}^{2}}{\sigma_{W}^{2}}\right), \\
R & \triangleq \frac{1}{2} \log \left(\frac{\sigma_{X}^{2} \sigma_{U}^{2}+\sigma_{X}^{2} \sigma_{W}^{2}+\sigma_{W}^{2} \sigma_{U}^{2}}{\left(\sigma_{X}^{2}+\sigma_{U}^{2}\right) \sigma_{W}^{2}}\right) .
\end{aligned}
$$

Draw $\left\lceil 2^{n R^{\prime}}\right\rceil \quad$ independent random $n$-vectors $\left\{\mathbf{Z}(1), \mathbf{Z}(2), \ldots, \mathbf{Z}\left(\left\lceil 2^{n R^{\prime}}\right\rceil\right)\right\}$ uniformly over the centered $n$-sphere of radius $r=\sqrt{n \sigma_{Z}^{2}}$. Assign these vectors to $\left\lfloor 2^{n(R+\delta)}\right\rfloor$ bins: the first $\left\lceil 2^{\left(R^{\prime}-R-\delta\right)}\right\rceil$ are assigned to bin 1 , the following $\left\lceil 2^{\left(R^{\prime}-R-\delta\right)}\right\rceil$ vectors are assigned to bin 2 , etc. More specifically, if $\mathcal{B}(m)$ denotes the set of vectors assigned to bin $m \in\left\{1, \ldots,\left\lfloor 2^{n(R+\delta)}\right\rfloor\right\}$, then

$$
\mathcal{B}(m)=\left\{\mathbf{Z}_{(m-1)\left\lceil 2^{\left(R^{\prime}-R-\delta\right)}\right\rceil+1}, \ldots, \mathbf{Z}_{m\left\lceil 2^{\left(R^{\prime}-R-\delta\right)}\right\rceil}\right\}
$$

for $m=1, \ldots,\left\lfloor 2^{n(R+\delta)}\right\rfloor-1$ and

$$
\mathcal{B}\left(\left\lfloor 2^{n(R+\delta)}\right\rfloor\right) \triangleq\left\{\mathbf{Z}_{\left(\left\lfloor 2^{n(R+\delta)}\right\rfloor-1\right)+1}, \ldots, \mathbf{Z}_{\left\lceil 2^{n R^{\prime}}\right\rceil}\right\} .
$$

The codebook $\mathcal{C} \triangleq\left\{\mathbf{Z}(1), \mathbf{Z}(2), \ldots, \mathbf{Z}\left(\left\lceil 2^{n R^{\prime}}\right\rceil\right)\right\}$.

2) Encoder: Given the source sequence $\mathbf{X}=\mathbf{x}$, the encoder looks for the codeword $\mathbf{z}^{*} \in \mathcal{C}$ that is closest to having the "correct" angle with $\mathbf{x}$ :

$$
\mathbf{z}^{*}=\underset{\mathbf{z} \in \mathcal{C}}{\arg \min }\left|\cos \varangle(\mathbf{x}, \mathbf{z})-\sqrt{1-2^{-2 R^{\prime}}}\right| .
$$

The encoder then sends $M=m^{*}$, where $m^{*}$ denotes the index of the bin containing $\mathbf{z}^{*}$. It also produces the reconstruction sequence $\hat{\mathbf{x}}_{\mathrm{e}}=\mathbf{z}^{*}+b \mathbf{x}$.

3) Decoder: Given $M=m^{*}$ and the side-information vector $\mathbf{Y}=\mathbf{y}$, the decoder chooses

$$
\hat{\mathbf{z}}=\underset{\mathbf{z} \in \mathcal{B}\left(m^{*}\right)}{\arg \min }\left|\cos \varangle(\mathbf{y}, \mathbf{z})-\sqrt{1-2^{-2\left(R^{\prime}-R\right)}}\right|,
$$

and produces the reconstruction sequence $\hat{\mathbf{x}}_{\mathrm{d}}=\hat{\mathbf{z}}+b \mathbf{y}$.

With probability 1 the argmins in (215) and (216) are unique.

\section{Analysis}

We fix $\epsilon>0$ sufficiently small such that

$$
(1-4 \epsilon) \sqrt{1-2^{-2\left(R^{\prime}-R\right)}}>\sqrt{1-2^{-2\left(R^{\prime}-R-\delta / 2\right)}},
$$

and define the following four events:

1) $\mathcal{E}_{\text {src }}$ : "The source and side information are atypical", i.e.,

$$
\begin{aligned}
\left|\frac{1}{n}\|\mathbf{X}\|^{2}-\sigma_{X}^{2}\right| & >\epsilon \sigma_{X}^{2} \quad \text { or } \\
\left|\frac{1}{n}\|\mathbf{Y}\|^{2}-\sigma_{Y}^{2}\right| & >\epsilon \sigma_{Y}^{2} \quad \text { or } \\
\left|\cos \varangle(\mathbf{X}, \mathbf{Y})-\rho_{X Y}\right| & >\epsilon \rho_{X Y}
\end{aligned}
$$

where $\rho_{X Y}$ denotes the correlation coefficient between $X$ and $Y$ :

$$
\rho_{X Y}=\sqrt{\frac{\sigma_{X}^{2}}{\sigma_{X}^{2}+\sigma_{U}^{2}}} .
$$


2) $\mathcal{E}_{\text {enc }}$ : "No codeword has a good angle with the source sequence", i.e.,

$$
\left|\cos \varangle\left(\mathbf{X}, \mathbf{Z}^{*}\right)-\sqrt{1-2^{-2 R^{\prime}}}\right|>\epsilon \sqrt{1-2^{-2 R^{\prime}}} .
$$

3) $\mathcal{E}_{\operatorname{dec} 1}$ : "The chosen codeword $\mathbf{Z}^{*}$ does not have the correct angle with the side-information sequence", i.e.,

$\left|\cos \varangle\left(\mathbf{Y}, \mathbf{Z}^{*}\right)-\sqrt{1-2^{-2\left(R^{\prime}-R\right)}}\right|>4 \epsilon \sqrt{1-2^{-2\left(R^{\prime}-R\right)}}$.

4) $\mathcal{E}_{\operatorname{dec} 2}:$ "The decoder does not find the correct codeword", i.e.,

$$
\hat{\mathbf{Z}} \neq \mathbf{Z}^{*} \text {. }
$$

Also, we define the event

$$
\mathcal{E} \triangleq \mathcal{E}_{\mathrm{src}} \cup \mathcal{E}_{\text {enc }} \cup \mathcal{E}_{\mathrm{dec} 1} \cup \mathcal{E}_{\mathrm{dec} 2} .
$$

Lemma 23:

$$
\lim _{n \rightarrow \infty} \operatorname{Pr}[\mathcal{E}]=0 .
$$

Proof: We note

$$
\begin{aligned}
\operatorname{Pr}[\mathcal{E}] \leq & \operatorname{Pr}\left[\mathcal{E}_{\mathrm{src}}\right]+\operatorname{Pr}\left[\mathcal{E}_{\mathrm{enc}} \mid \mathcal{E}_{\mathrm{src}}^{c}\right]+\operatorname{Pr}\left[\mathcal{E}_{\mathrm{dec} 1} \mid \mathcal{E}_{\mathrm{src}}^{c} \cap \mathcal{E}_{\mathrm{enc}}^{c}\right] \\
& +\operatorname{Pr}\left[\mathcal{E}_{\mathrm{dec} 2} \mid \mathcal{E}_{\mathrm{src}}^{c} \cap \mathcal{E}_{\mathrm{enc}}^{c}\right]
\end{aligned}
$$

In the following we show that each term on the RHS of (224) tends to zero as the blocklength $n$ tends to infinity. The first limit

$$
\lim _{n \rightarrow \infty} \operatorname{Pr}\left[\mathcal{E}_{\text {src }}\right]=0
$$

follows directly from the weak law of large numbers. The second limit

$$
\lim _{n \rightarrow \infty} \operatorname{Pr}\left[\mathcal{E}_{\mathrm{enc}} \mid \mathcal{E}_{\mathrm{src}}^{c}\right]=0
$$

can be shown following the same steps as in the proof of Limit (134) in [20]. The third limit

$$
\lim _{n \rightarrow \infty} \operatorname{Pr}\left[\mathcal{E}_{\mathrm{dec} 1} \mid \mathcal{E}_{\mathrm{src}}^{c} \cap \mathcal{E}_{\mathrm{enc}}^{c}\right]=0
$$

is proved as follows. We have

$$
\cos \varangle\left(\mathbf{Y}, \mathbf{Z}^{*}\right)=\cos \varangle(\mathbf{X}, \mathbf{Y}) \cos \varangle\left(\mathbf{X}, \mathbf{Z}^{*}\right)+\frac{\left\langle\mathbf{Y}^{\perp}, \mathbf{Z}^{* \perp}\right\rangle}{\|\mathbf{Y}\|\left\|\mathbf{Z}^{*}\right\|}
$$

where $\mathbf{Y}^{\perp}$ and $\mathbf{Z}^{* \perp}$ denote the components of $\mathbf{Y}$ and $\mathbf{Z}$ that are orthogonal to $\mathbf{X}$ :

$$
\begin{aligned}
\mathbf{Y}^{\perp} & \triangleq \mathbf{Y}-\frac{\langle\mathbf{X}, \mathbf{Y}\rangle}{\|\mathbf{X}\|^{2}} \mathbf{X} \\
& =\mathbf{Y}-\cos \varangle(\mathbf{X}, \mathbf{Y})\|\mathbf{Y}\| \frac{\mathbf{X}}{\|\mathbf{X}\|},
\end{aligned}
$$

and

$$
\begin{aligned}
\mathbf{Z}^{* \perp} & \triangleq \mathbf{Z}^{*}-\frac{\left\langle\mathbf{X}, \mathbf{Z}^{*}\right\rangle}{\|\mathbf{X}\|^{2}} \mathbf{X} \\
& =\mathbf{Z}^{*}-\cos \varangle\left(\mathbf{X}, \mathbf{Z}^{*}\right)\left\|\mathbf{Z}^{*}\right\| \frac{\mathbf{X}}{\|\mathbf{X}\|} .
\end{aligned}
$$

Let $t_{X Z^{*}}$ satisfy

$$
t_{X Z^{*}} \in\left[(1-\epsilon) \sqrt{2^{-2 R^{\prime}}},(1+\epsilon) \sqrt{2^{-2 R^{\prime}}}\right]
$$

and let $\mathbf{x}$ and $\mathbf{y}$ be vectors in $\mathbb{R}^{n}$ satisfying

$$
\begin{aligned}
\left|\frac{1}{n}\|\mathbf{x}\|^{2}-\sigma_{X}^{2}\right| & \leq \epsilon \sigma_{X}^{2} \\
\left|\frac{1}{n}\|\mathbf{y}\|^{2}-\sigma_{Y}^{2}\right| & \leq \epsilon \sigma_{Y}^{2} \sigma_{Y}^{2} \\
\left|\cos \varangle(\mathbf{x}, \mathbf{y})-\rho_{X Y}\right| & \leq \epsilon \rho_{X Y} .
\end{aligned}
$$

Then, conditional on events

$$
\mathcal{E}_{\text {src }}^{c}, \quad \mathcal{E}_{\text {enc }}^{c}, \quad \mathbf{X}=\mathbf{x}, \quad \mathbf{Y}=\mathbf{y}, \quad \cos \varangle\left(\mathbf{X}, \mathbf{Z}^{*}\right)=t_{X Z^{*}},
$$

by (233) and (234c), we have

$$
\begin{aligned}
\cos \varangle(\mathbf{X}, \mathbf{Y}) \cos \varangle\left(\mathbf{X}, \mathbf{Z}^{*}\right) & \leq(1+\epsilon) \rho_{X Y}(1+\epsilon) \sqrt{2^{-2 R^{\prime}}} \\
& \stackrel{(a)}{\leq} \sqrt{1-2^{-\left(R^{\prime}-R\right)}}(1+3 \epsilon)
\end{aligned}
$$

and

$$
\begin{aligned}
\cos \varangle(\mathbf{X}, \mathbf{Y}) \cos \varangle\left(\mathbf{X}, \mathbf{Z}^{*}\right) & \geq(1-\epsilon) \rho_{X Y}(1-\epsilon) \sqrt{2^{-2 R^{\prime}}} \\
& \stackrel{(a)}{\geq} \sqrt{1-2^{-\left(R^{\prime}-R\right)}}(1-3 \epsilon),
\end{aligned}
$$

where Inequalities $(a)$ follow because

$$
\rho_{X Y} \cdot \sqrt{1-2^{-2 R^{\prime}}}=\sqrt{1-2^{-\left(R^{\prime}-R\right)}}
$$

and because $\epsilon \in(0,1)$. Moreover, conditional on the events in (235), the vector $\mathbf{Z}^{* \perp}$ is uniformly distributed over a centered $(n-1)$-dimensional sphere of radius $\sigma_{Z}^{2}\left(1-t_{X Z^{*}}^{2}\right)$, and thus Limit (238) on top of the next page follows by Lemmas 19 and 22.

We can combine Limit (238) and Inequalities (236) to obtain the limit (239) on top of the next page. If in (239) we take the expectation with respect to $\mathbf{X}, \mathbf{Y}$, and $\cos \varangle\left(\mathbf{X}, \mathbf{Z}^{*}\right)$ (but keep the conditioning on events $\mathcal{E}_{\text {src }}^{c}$ and $\mathcal{E}_{\text {enc }}^{c}$ ), we obtain the desired third limit (227).

We finally prove the fourth limit

$$
\lim _{n \rightarrow \infty} \operatorname{Pr}\left[\mathcal{E}_{\mathrm{dec} 2} \mid \mathcal{E}_{\mathrm{src}}^{c} \cap \mathcal{E}_{\mathrm{enc}}^{c}\right]=0 .
$$

To this end, we define event $\mathcal{E}_{2}$ as

$$
\cos \varangle\left(\mathbf{Y}, \mathbf{Z}^{\prime}\right)<\sqrt{1-2^{-2\left(R^{\prime}-R-\delta / 2\right)}}, \quad \forall \mathbf{Z}^{\prime} \in\left(\mathcal{B}(M) \backslash \mathbf{Z}^{*}\right) .
$$

Recalling the decoding rule in (216) and the definition of event $\mathcal{E}_{\mathrm{dec} 1}$ in (221), we see that when $\mathcal{E}_{\mathrm{dec} 1}^{c}$ and $\mathcal{E}_{2}$ occur simultaneously, then by condition (217) the decoder finds the correct codeword $\hat{\mathbf{Z}}=\mathbf{Z}^{*}$. Therefore,

$$
\operatorname{Pr}\left[\mathcal{E}_{\mathrm{dec} 2} \mid \mathcal{E}_{\mathrm{src}}^{c}, \mathcal{E}_{\mathrm{enc}}^{c}\right] \leq 1-\operatorname{Pr}\left[\mathcal{E}_{\mathrm{dec} 1}^{c} \cap \mathcal{E}_{2} \mid \mathcal{E}_{\mathrm{src}}^{c}, \mathcal{E}_{\mathrm{enc}}^{c}\right],
$$

and thus (227) and the limit

$$
\lim _{n \rightarrow \infty} \operatorname{Pr}\left[\mathcal{E}_{2}^{c} \mid \mathcal{E}_{\mathrm{src}}^{c}, \mathcal{E}_{\text {enc }}^{c}\right]=0
$$

establish (240).

We now prove (243). For each $m \in\left\{1, \ldots,\left\lfloor 2^{n(R+\delta)}\right\rfloor\right\}$, we index the vectors in the $m$-th bin from 1 to $|\mathcal{B}(m)|$ and we shall refer to the $k$-th vector in this $m$-th bin by $\mathbf{Z}_{m, k}$. Let $K^{*}$ be the index of $\mathbf{Z}^{*}$, i.e., $\mathbf{Z}_{M, K^{*}}=\mathbf{Z}^{*}$. By the symmetry of the code construction and the encoding rule, the probability $\operatorname{Pr}\left[\mathcal{E}^{c} \mid \mathcal{E}_{\text {src }}^{c}, \mathcal{E}_{\text {enc }}^{c}, M=m, K^{*}=k\right]$ does 


$$
\begin{aligned}
& \lim _{n \rightarrow \infty} \operatorname{Pr}\left[\left|\left\langle\mathbf{y}^{\perp}, \mathbf{Z}^{* \perp}\right\rangle\right| \leq \epsilon \sqrt{1-2^{-2\left(R^{\prime}-R\right)}}\|\mathbf{y}\| \sqrt{\sigma_{Z}^{2}} \mid \mathbf{X}=\mathbf{x}, \mathbf{Y}=\mathbf{y}, \cos \varangle\left(\mathbf{X}, \mathbf{Z}^{*}\right)=t_{X Z^{*}}\right]=1 \\
& \lim _{n \rightarrow \infty} \operatorname{Pr}\left[\left|\cos \varangle\left(\mathbf{Y}, \mathbf{Z}^{*}\right)-\sqrt{1-2^{-2\left(R^{\prime}-R\right)}}\right| \leq 4 \epsilon \sqrt{1-2^{-2\left(R^{\prime}-R\right)}} \mid \mathcal{E}_{\text {src }}^{c}, \mathcal{E}_{\text {enc }}^{c}, \mathbf{X}=\mathbf{x}, \mathbf{Y}=\mathbf{y}, \cos \varangle\left(\mathbf{X}, \mathbf{Z}^{*}\right)=t_{X Z^{*}}\right]=1 \\
& \operatorname{Pr}\left[\bigcup_{k=2}^{|\mathcal{B}(1)|}\left(\cos \varangle\left(\mathbf{Y}, \mathbf{Z}_{1, k}\right) \geq \sqrt{1-2^{-2\left(R^{\prime}-R-\delta / 2\right)}}\right) \mid \mathbf{X}=\mathbf{x}, M=1, K^{*}=1, \mathcal{E}_{\text {src }}^{c}, \mathcal{E}_{\text {enc }}^{c}\right] \\
& =1-\prod_{k=2}^{|\mathcal{B}(1)|}\left(1-\operatorname{Pr}\left[\cos \varangle\left(\mathbf{Y}, \mathbf{Z}_{1, k}\right) \geq \sqrt{1-2^{-2\left(R^{\prime}-R-\delta / 2\right)}} \mid \mathbf{X}=\mathbf{x}, M=1, K^{*}=1, \mathcal{E}_{\text {src }}^{c}, \mathcal{E}_{\text {enc }}^{c}\right]\right) \\
& <1-\left(1-\frac{2 C_{n}\left(\arccos \left(\sqrt{1-2^{-2\left(R^{\prime}-R-\delta / 2\right)}}\right)\right)}{C_{n}(\pi)}\right)^{|\mathcal{B}(1)|-1} \\
& \leq 1-\left(1-\frac{2 C_{n}\left(\arccos \left(\sqrt{1-2^{-2\left(R^{\prime}-R-\delta / 2\right)}}\right)\right)}{C_{n}(\pi)}\right)^{2^{n\left(R^{\prime}-R-\delta\right)}}
\end{aligned}
$$

not depend on the values $m$ and $k$. We therefore, assume in the following that $M=1$ and $K^{*}=1$. If we additionally condition on $\mathbf{X}=\mathbf{x}$ and on $\cos \varangle\left(\mathbf{X}, \mathbf{Z}^{*}\right)=t_{X Z^{*}}>0$, the vectors $\mathbf{Z}_{1,2}, \ldots, \mathbf{Z}_{1,|\mathcal{B}(1)|}$ (i.e., the vectors in bin 1 that are not $\mathbf{Z}^{*}$ ) are independent and uniformly distributed over the centered $n$-sphere of radius $\sqrt{n \sigma_{Z}^{2}}$ without the spherical cap of half-angle $\arccos \left(t_{X Z^{*}}\right)$ centered at $\mathbf{x}$. Thus, $\frac{2}{C_{n}(\pi)}$ is an upper bound on the conditional density of the normalized vectors $\frac{1}{\sqrt{n \sigma_{Z}^{2}}} \mathbf{Z}_{1,2}, \ldots, \frac{1}{\sqrt{n \sigma_{Z}^{2}}} \mathbf{Z}_{1,|\mathcal{B}(1)|}$ on the centered unit $n$-sphere. Applying Lemma 19, we therefore obtain Inequality (245) shown on top of this page. We note that for any $\gamma \in[0,1]$

$$
0 \leq\left(1-\frac{2 C_{n}(\arccos (\gamma))}{C_{n}(\pi)}\right) \leq 1
$$

and hence the mapping $t \mapsto\left(1-\frac{2 C_{n}(\arccos (\gamma))}{C_{n}(\pi)}\right)^{t}$ is decreasing in $t>0$. Therefore, since

$$
|\mathcal{B}(1)|-1<2^{n\left(R^{\prime}-R-\delta\right)}
$$

we further obtain (246). If now we take the expectation with respect to $\mathbf{X}, M$, and $K^{*}$ (but keep the conditioning on $\mathcal{E}_{\text {src }}^{c}$ and $\mathcal{E}_{\text {enc }}^{c}$ ), (246) results in

$$
\begin{aligned}
& \operatorname{Pr}\left[\mathcal{E}_{2} \mid \mathcal{E}_{\mathrm{src}}^{c}, \mathcal{E}_{\mathrm{enc}}^{c}\right] \\
& \quad<1-\left(1-\frac{2 C_{n}\left(\arccos \sqrt{1-2^{-2\left(R^{\prime}-R-\frac{\delta}{2}\right)}}\right)}{C_{n}(\pi)}\right)^{2^{n\left(R^{\prime}-R-\delta\right)}}
\end{aligned}
$$

The desired limit (243) follows by (249) and by Lemma 21 . In fact, applying Lemma 21 to

$$
\eta_{2}=R^{\prime}-R-\delta
$$

and to the function

$$
f: n \rightarrow \frac{2 C_{n}\left(\arccos \left(\sqrt{1-2^{-2\left(R^{\prime}-R-\delta / 2\right)}}\right)\right)}{C_{n}(\pi)},
$$

we obtain that the right-hand side of (249) tends to 1 as $n$ tends to infinity because

$$
\begin{aligned}
\eta_{1} & \triangleq-\lim _{n \rightarrow \infty} \frac{1}{n} \log \left(\frac{2 C_{n}\left(\arccos \left(\sqrt{1-2^{-2\left(R^{\prime}-R-\delta / 2\right)}}\right)\right)}{C_{n}(\pi)}\right) \\
& =R^{\prime}-R-\delta / 2 \\
& >\eta_{2} .
\end{aligned}
$$

Here, the equality holds by Lemma 20 and because the factor 2 in the logarithm does not change the limit, and the inequality holds by (250) and because $\delta>0$.

This concludes the proof of limit (243) and thus of the fourth limit (240). Combining finally (224) with (225)-(227) and (240) establishes the proof of the lemma.

We can now bound the expected distortions of our scheme. We have

$$
\begin{aligned}
\mathrm{E}\left[d_{\mathrm{d}}^{(n)}\left(\mathbf{X}, \hat{\mathbf{X}}_{\mathrm{d}}\right)\right]= & \operatorname{Pr}\left[\mathcal{E}^{c}\right] \mathrm{E}\left[d_{\mathrm{d}}^{(n)}\left(\mathbf{X}, \hat{\mathbf{X}}_{\mathrm{d}}\right) \mid \mathcal{E}^{c}\right] \\
& +\operatorname{Pr}[\mathcal{E}] \mathrm{E}\left[d_{\mathrm{d}}^{(n)}\left(\mathbf{X}, \hat{\mathbf{X}}_{\mathrm{d}}\right) \mid \mathcal{E}\right],
\end{aligned}
$$

and

$$
\begin{aligned}
\mathrm{E}\left[d_{\mathrm{e}}^{(n)}\left(\hat{\mathbf{X}}_{\mathrm{d}}, \hat{\mathbf{X}}_{\mathrm{e}}\right)\right]= & \operatorname{Pr}\left[\mathcal{E}^{c}\right] \mathrm{E}\left[d_{\mathrm{e}}^{(n)}\left(\hat{\mathbf{X}}_{\mathrm{d}}, \hat{\mathbf{X}}_{\mathrm{e}}\right) \mid \mathcal{E}^{c}\right] \\
& +\operatorname{Pr}[\mathcal{E}] \mathrm{E}\left[d_{\mathrm{e}}^{(n)}\left(\hat{\mathbf{X}}_{\mathrm{d}}, \hat{\mathbf{X}}_{\mathrm{e}}\right) \mid \mathcal{E}\right] .
\end{aligned}
$$

The decoder-side distortion satisfies

$$
\begin{aligned}
d_{\mathrm{d}}^{(n)}\left(\mathbf{x}, \hat{\mathbf{x}}_{\mathrm{d}}\right) & =\frac{1}{n}\left\|\mathbf{x}-\mathbf{z}^{*}-b \mathbf{y}\right\|^{2} \\
& \leq \frac{3}{n}\|\mathbf{x}\|^{2}+\frac{3}{n}\left\|\mathbf{z}^{*}\right\|^{2}+\frac{3}{n} b^{2}\|\mathbf{y}\|^{2},
\end{aligned}
$$

where the inequality holds by the Cauchy-Schwarz Inequality and because an arithmetic mean of two nonnegative numbers cannot be smaller than it's geometric mean. 
Therefore,

$$
\begin{aligned}
\operatorname{Pr} & {[\mathcal{E}] \mathrm{E}\left[d_{\mathrm{d}}^{(n)}\left(\mathbf{X}, \hat{\mathbf{X}}_{\mathrm{d}}\right) \mid \mathcal{E}\right] } \\
\leq & \frac{3}{n} \operatorname{Pr}[\mathcal{E}] \mathrm{E}\left[\|\mathbf{X}\|^{2}+\left\|\mathbf{Z}^{*}\right\|^{2}+b^{2}\|\mathbf{Y}\|^{2} \mid \mathcal{E}\right] \\
= & \frac{3}{n} \mathrm{E}\left[\|\mathbf{X}\|^{2}+\left\|\mathbf{Z}^{*}\right\|^{2}+b^{2}\|\mathbf{Y}\|^{2}\right] \\
& -\frac{3}{n} \operatorname{Pr}\left[\mathcal{E}^{c}\right] \mathrm{E}\left[\|\mathbf{X}\|^{2}+\left\|\mathbf{Z}^{*}\right\|^{2}+b^{2}\|\mathbf{Y}\|^{2} \mid \mathcal{E}^{c}\right] \\
\leq & 3\left(\sigma_{X}^{2}+\sigma_{Z}^{2}+b^{2}\left(\sigma_{X}^{2}+\sigma_{U}^{2}\right)\right) \\
& -3\left(\sigma_{X}^{2}(1-\epsilon)+\sigma_{Z}^{2}+b^{2}\left(\sigma_{X}^{2}+\sigma_{U}^{2}\right)(1-\epsilon)\right) \operatorname{Pr}\left[\mathcal{E}^{c}\right] \\
\leq & 3\left(\sigma_{X}^{2}+\sigma_{Z}^{2}+b^{2}\left(\sigma_{X}^{2}+\sigma_{U}^{2}\right)\right)\left(1-(1-\epsilon) \operatorname{Pr}\left[\mathcal{E}^{c}\right]\right) .
\end{aligned}
$$

In the event $\mathcal{E}^{c}$, we can derive a bound on the decoder-side distortion $d_{\mathrm{d}}^{(n)}\left(\mathbf{x}, \hat{\mathbf{x}}_{\mathrm{d}}\right)$ that is tighter than (257):

$$
\begin{aligned}
d_{\mathrm{d}}^{(n)} & \left(\mathbf{x}, \hat{\mathbf{x}}_{\mathrm{d}}\right) \\
= & \frac{1}{n}\left\|\mathbf{x}-\mathbf{z}^{*}-b \mathbf{y}\right\|^{2} \\
= & \frac{1}{n}\|\mathbf{x}\|^{2}+\frac{1}{n}\left\|\mathbf{z}^{*}\right\|^{2}+\frac{b^{2}}{n}\|\mathbf{y}\|^{2} \\
& -\frac{2}{n}\left\langle\mathbf{x}, \mathbf{z}^{*}\right\rangle-\frac{2 b}{n}\langle\mathbf{x}, \mathbf{y}\rangle+\frac{2 b}{n}\left\langle\mathbf{z}^{*}, \mathbf{y}\right\rangle \\
\leq & (1+\epsilon) \sigma_{X}^{2}+\sigma_{Z}^{2}+(1+\epsilon) b^{2}\left(\sigma_{X}^{2}+\sigma_{U}^{2}\right) \\
& -2(1-\epsilon)^{2} a \sigma_{X}^{2}-2(1-\epsilon)^{3} b \sigma_{X}^{2} \\
& +2(1+\epsilon)(1+4 \epsilon) a b \sigma_{X}^{2} \\
\leq & \left(1+a^{2}+b^{2}-2 a-2 b+2 a b\right) \sigma_{X}^{2}+a^{2} \sigma_{W}^{2}+b^{2} \sigma_{U}^{2} \\
& +\epsilon\left(\sigma_{X}^{2}+b^{2}\left(\sigma_{X}^{2}+\sigma_{U}^{2}\right)+4 a \sigma_{X}^{2}+6 b \sigma_{X}^{2}+10 a b \sigma_{X}^{2}\right) \\
& +8 \epsilon^{2} a b \sigma_{X}^{2}+2 \epsilon^{3} b \sigma_{X}^{2} \\
\leq & D_{\mathrm{d}} \\
& +\epsilon\left(\sigma_{X}^{2}+b^{2}\left(\sigma_{X}^{2}+\sigma_{U}^{2}\right)+4 a \sigma_{X}^{2}+8 b \sigma_{X}^{2}+18 a b \sigma_{X}^{2}\right)
\end{aligned}
$$

where the first inequality follows from the definition of the event $\mathcal{E}^{c}$, the second by throwing away some negative $\epsilon$-terms, and the third from Condition (209) and because $\epsilon<1$. Since $\operatorname{Pr}\left[\mathcal{E}^{c}\right] \leq 1$, we thus have:

$$
\begin{aligned}
& \operatorname{Pr}\left[\mathcal{E}^{c}\right] \mathrm{E}\left[d_{\mathrm{e}}^{(n)}\left(\hat{\mathbf{X}}_{\mathrm{d}}, \hat{\mathbf{X}}_{\mathrm{e}}\right) \mid \mathcal{E}^{c}\right] \\
& \leq D_{d}+\epsilon\left(\sigma_{X}^{2}+b^{2}\left(\sigma_{X}^{2}+\sigma_{U}^{2}\right)+4 a \sigma_{X}^{2}+8 b \sigma_{X}^{2}+18 a b \sigma_{X}^{2}\right) .
\end{aligned}
$$

Combining (254), (261), and (267), we obtain

$$
\begin{aligned}
& \mathrm{E}\left[d_{\mathrm{d}}^{(n)}\left(\mathbf{X}, \hat{\mathbf{X}}_{\mathrm{d}}\right)\right] \\
& \quad \leq D_{\mathrm{d}}+3\left(\sigma_{X}^{2}+\sigma_{Z}^{2}+b^{2} \sigma_{Y}^{2}\right)\left(1-(1+\epsilon) \operatorname{Pr}\left[\mathcal{E}^{c}\right]\right) \\
& \quad+\epsilon\left(\sigma_{X}^{2}+b^{2}\left(\sigma_{X}^{2}+\sigma_{U}^{2}\right)+4 a \sigma_{X}^{2}+8 b \sigma_{X}^{2}+18 a b \sigma_{X}^{2}\right)
\end{aligned}
$$

Similarly, we have for the encoder-side distortion:

$$
\begin{aligned}
d_{\mathrm{e}}^{(n)}\left(\mathbf{x}, \hat{\mathbf{x}}_{\mathrm{d}}\right) & =\frac{1}{n}\|b \mathbf{y}-b \mathbf{x}\|^{2} \\
& \leq \frac{2}{n} b^{2}\|\mathbf{y}\|^{2}+\frac{2}{n} b^{2}\|\mathbf{x}\|^{2},
\end{aligned}
$$

and thus,

$$
\begin{aligned}
\operatorname{Pr}[\mathcal{E}] & \mathrm{E}\left[d_{\mathrm{e}}^{(n)}\left(\mathbf{X}_{\mathrm{d}}, \hat{\mathbf{X}}_{\mathrm{e}}\right) \mid \mathcal{E}\right] \\
\leq & \frac{2}{n} \mathrm{E}\left[b^{2}\|\mathbf{Y}\|^{2}+b^{2}\|\mathbf{X}\|^{2}\right] \\
& -\frac{2}{n} \operatorname{Pr}\left[\mathcal{E}^{c}\right] \mathrm{E}\left[b^{2}\|\mathbf{Y}\|^{2}+b^{2}\|\mathbf{X}\|^{2} \mid \mathcal{E}^{c}\right] \\
\leq & 2\left(b^{2}\left(\sigma_{X}^{2}+\sigma_{U}^{2}\right)+b^{2} \sigma_{X}^{2}\right)\left(1-(1-\epsilon) \operatorname{Pr}\left[\mathcal{E}^{c}\right]\right) .
\end{aligned}
$$

Moreover, in the event $\mathcal{E}^{c}$ we can derive a bound on the encoder-side distortion $d_{\mathrm{e}}^{(n)}\left(\hat{\mathbf{x}}_{\mathrm{d}}, \hat{\mathbf{x}}_{\mathrm{e}}\right)$ that is tighter than (271):

$$
\begin{aligned}
d_{\mathrm{e}}^{(n)}\left(\hat{\mathbf{x}}_{\mathrm{d}}, \hat{\mathbf{x}}_{\mathrm{e}}\right)= & \frac{1}{n}\|b \mathbf{y}-b \mathbf{x}\|^{2} \\
= & \frac{1}{n} b^{2}\left(\|\mathbf{x}\|^{2}+\|\mathbf{y}\|^{2}-2\langle\mathbf{x}, \mathbf{y}\rangle\right) \\
\leq & (1+\epsilon) b^{2} \sigma_{X}^{2}+(1+\epsilon) b^{2}\left(\sigma_{X}^{2}+\sigma_{U}^{2}\right) \\
& -2 b^{2}(1-\epsilon)^{3} \sigma_{X}^{2} \\
\leq & b^{2} \sigma_{U}^{2}+\epsilon b^{2}\left(8 \sigma_{X}^{2}+\sigma_{U}^{2}\right)+\epsilon^{3} b^{2} \sigma_{X}^{2} \\
\leq & D_{\mathrm{e}}+\epsilon b^{2}\left(9 \sigma_{X}^{2}+\sigma_{U}^{2}\right)
\end{aligned}
$$

where the last inequality follows by Assumption (210) and because $\epsilon<1$. Since $\operatorname{Pr}\left[\mathcal{E}^{c}\right] \leq 1$, we thus have

$$
\operatorname{Pr}\left[\mathcal{E}^{c}\right] \mathrm{E}\left[d_{\mathrm{e}}^{(n)}\left(\hat{\mathbf{X}}_{\mathrm{d}}, \hat{\mathbf{X}}_{\mathrm{e}}\right) \mid \mathcal{E}^{c}\right] \leq D_{\mathrm{e}}+\epsilon b^{2}\left(9 \sigma_{X}^{2}+\sigma_{U}^{2}\right) .
$$

Combining finally (255), (273), and (279), we obtain

$$
\begin{aligned}
& \mathrm{E}\left[d_{\mathrm{e}}^{(n)}\left(\mathbf{X}_{\mathrm{d}}, \hat{\mathbf{X}}_{\mathrm{e}}\right)\right] \\
& \quad \leq D_{\mathrm{e}}+2\left(b^{2} \sigma_{Y}^{2}+b^{2} \sigma_{X}^{2}\right)\left(1-(1-\epsilon) \operatorname{Pr}\left[\mathcal{E}^{c}\right]\right) \\
& \quad+\epsilon b^{2}\left(9 \sigma_{X}^{2}+\sigma_{U}^{2}\right) .
\end{aligned}
$$

Recall that the rate of our scheme is smaller than $R+\delta$ and that $\epsilon, \delta>0$ can be chosen arbitrarily close to 0 . Therefore, from (269), (280), and Lemma 23 we conclude that when $a, \sigma_{W}^{2}>0$ and $b \geq 0$ satisfy (209) and (210), then our scheme can achieve the triple

$$
\left(R=\frac{1}{2} \log \left(\frac{\sigma_{X}^{2} \sigma_{U}^{2}+\sigma_{X}^{2} \sigma_{W}^{2}+\sigma_{U}^{2} \sigma_{W}^{2}}{\left(\sigma_{X}^{2}+\sigma_{U}^{2}\right) \sigma_{W}^{2}}\right), D_{\mathrm{d}}, D_{\mathrm{e}}\right) .
$$

This establishes Proposition 11.

\section{APPENDIX D}

\section{THE CARDinALITY BOUND ON $\mathcal{U}$}

To prove the cardinality bound (162) on $\mathcal{U}$, we shall need the following variation on Carathéodory's theorem.

Lemma 24: Any point on the boundary of the convex hull of a compact set in $\mathbb{R}^{d}$ can be expressed as a convex combination of $d$ or fewer points in the set.

Proof: Let $\mathcal{S}$ be a compact subset of $\mathbb{R}^{d}$, and let $\mathbf{x}$ be a boundary point of its convex hull $\operatorname{conv}(\mathcal{S})$. Since $\mathbf{x}$ is in the 
convex hull of $\mathcal{S}$, it follows from Carathéodory's theorem that there exist $d+1$ or fewer points

$$
\mathbf{x}_{1}, \ldots, \mathbf{x}_{v} \in \mathcal{S}, \quad v \leq d+1
$$

and positive coefficients summing to 1

$$
\lambda_{1}, \ldots, \lambda_{v}>0, \quad \sum_{i=1}^{v} \lambda_{i}=1
$$

such that

$$
\mathbf{x}=\sum_{i=1}^{v} \lambda_{i} \mathbf{x}_{i}
$$

We shall show that, in fact, of these $v$ points, we can find $d$ or fewer points whose convex combination is $\mathbf{x}$.

Since $\mathbf{x}$ is on the boundary of $\operatorname{conv}(\mathcal{S})$, there exists a hyperplane $\mathcal{H}$ that supports $\operatorname{conv}(\mathcal{S})$ at $\mathbf{x}$. Thus,

$$
\mathcal{H}=\left\{\xi \in \mathbb{R}^{d}: \mathbf{c}^{\top} \xi=\mathbf{c}^{\top} \mathbf{x}\right\}
$$

for some vector $\mathbf{c} \in \mathbb{R}^{d}$ and

$$
\mathbf{c}^{\top} \mathbf{x}=\max _{\tilde{\mathbf{x}} \in \operatorname{conv}(\mathcal{S})} \mathbf{c}^{\top} \mathbf{x}
$$

So

$$
\mathbf{c}^{\top} \mathbf{x} \geq \mathbf{c}^{\top} \mathbf{x}_{i}, \quad i=1, \ldots, v .
$$

We shall next show that the points $\mathbf{x}_{1}, \ldots, \mathbf{x}_{v}$ are in $\mathcal{H}$. To that end we note that by (284)

$$
\begin{aligned}
0 & =\mathbf{c}^{\top}\left(\mathbf{x}-\sum_{i=1}^{v} \lambda_{i} \mathbf{x}_{i}\right) \\
& =\sum_{i=1}^{v} \lambda_{i} \mathbf{c}^{\top} \mathbf{x}-\sum_{i=1}^{v} \lambda_{i} \mathbf{c}^{\top} \mathbf{x}_{i} \\
& =\sum_{i=1}^{v} \lambda_{i}\left(\mathbf{c}^{\top} \mathbf{x}-\mathbf{c}^{\top} \mathbf{x}_{i}\right)
\end{aligned}
$$

where the second equality holds because the $\lambda$ 's sum to 1 (283). Since the $\lambda$ 's are all positive, it follows from (286) that all the terms on the RHS are nonnegative. Since they sum to zero, they must all be zero. And since the $\lambda$ 's are positive, we conclude that

$$
\mathbf{c}^{\top} \mathbf{x}_{i}=\mathbf{c}^{\top} \mathbf{x}, \quad i \in\{1, \ldots, v\}
$$

and the vectors $\mathbf{x}_{i}$ are all in $\mathcal{H}$. The vector $\mathbf{x}$ can thus be written as a convex combination of the $v$ vectors in $\mathbf{x}_{1}, \ldots, \mathbf{x}_{v}$ in $\mathcal{H}$. Since $\mathcal{H}$ is $(d-1)$-dimensional, it follows from Carathéodory's theorem that $\mathbf{x}$ is in fact a convex combination of $d$ or fewer of the vectors $\mathbf{x}_{1}, \ldots, \mathbf{x}_{v}$.

The cardinality bound on $\mathcal{U}$ can now be proved as follows. Proof of the Cardinality Bound on $\mathcal{U}$ in Proposition 15: Let the discrete random variables $U$ and $Z$ over the alphabets $\mathcal{U}$ and $\mathcal{Z}$, the function $\phi: \mathcal{Y} \times \mathcal{Z} \rightarrow \hat{\mathcal{X}}_{\mathrm{d}}$, and the function $\psi: \mathcal{X} \times \mathcal{Z} \times \mathcal{U} \rightarrow \hat{\mathcal{X}}_{\mathrm{e}}$ satisfy (159) and (160). We shall exhibit a random variable $\tilde{U}$ over the alphabet

$$
\tilde{\mathcal{U}} \triangleq\{1, \ldots, K\}
$$

and a function $\tilde{\psi}: \mathcal{X} \times \mathcal{Z} \times \tilde{\mathcal{U}} \rightarrow \hat{\mathcal{X}}_{\mathrm{e}}$ satisfying

$$
\tilde{U} \multimap(X, Z) \multimap Y
$$

and the $K$ distortion constraints

$$
\mathrm{E}\left[d_{k}(X, \phi(Y, Z), \tilde{\psi}(X, Z, \tilde{U}))\right] \leq D_{k}, \quad k \in\{1, \ldots, K\} .
$$

Since the Markov conditions (159) and (289) imply

$$
(\tilde{U}, Z) \multimap X \multimap-Y \text {, }
$$

this will allow us to replace $U$ and $\psi$ with $\tilde{U}$ and $\tilde{\psi}$ and thus conclude the proof.

To describe $\tilde{U}$ and $\tilde{\psi}$, we need some definitions. For each pair $(x, z) \in \mathcal{X} \times \mathcal{Z}$ and each $k \in\{1, \ldots, K\}$, define

$$
\begin{aligned}
D_{k}^{(x, z)} & =\operatorname{Pr}\left[d_{k}(X, \phi(Y, Z), \psi(X, Z, U)) \mid(X, Z)=(x, z)\right] \\
& =\mathrm{E}\left[d_{k}(x, \phi(Y, z), \psi(x, z, U))\right],
\end{aligned}
$$

where the expectation is, by (159), with respect to $P_{U \mid X Z}(\cdot \mid x, z) P_{Y \mid X}(\cdot \mid x)$. Define also the vector-valued function

$$
\begin{aligned}
h^{(x, z)}: \mathcal{U} & \rightarrow \mathbb{R}_{+}^{K} \\
u & \mapsto\left(\begin{array}{c}
\mathrm{E}\left[d_{1}(x, \phi(Y, z), \psi(x, z, u))\right] \\
\vdots \\
\mathrm{E}\left[d_{K}(x, \phi(Y, z), \psi(x, z, u))\right]
\end{array}\right)
\end{aligned}
$$

where the expectation is with respect to $P_{Y \mid X}(\cdot \mid x)$. Let $\mathcal{S}^{(x, z)}$ denote the image of $h^{(x, z)}$ :

$$
\mathcal{S}^{(x, z)} \triangleq\left\{\mathbf{s} \in \mathbb{R}_{+}^{K}: \mathbf{s}=h^{(x, z)}(u) \text { for some } u \in \mathcal{U}\right\} .
$$

By definitions (292)-(294)

$$
\left(\begin{array}{c}
D_{1}^{(x, z)} \\
\vdots \\
D_{K}^{(x, z)}
\end{array}\right) \in \operatorname{conv}\left(\mathcal{S}^{(x, z)}\right)
$$

and, consequently, there exists a point

$$
\overline{\mathbf{s}}^{(x, z)}=\left(\begin{array}{c}
\bar{s}_{1}^{(x, z)} \\
\vdots \\
\bar{s}_{K}^{(x, z)}
\end{array}\right)
$$

on the boundary of $\operatorname{conv}\left(\mathcal{S}^{(x, z)}\right)$ with

$$
\bar{s}_{k}^{(x, z)} \leq D_{k}^{(x, z)}, \quad k \in\{1, \ldots, K\}
$$

Since $\mathcal{S}^{(x, z)}$ is compact (it contains at most $\left|\hat{\mathcal{X}}_{\mathrm{e}}\right|$ points because $h^{(x, z)}(u)$ depends on $u$ only via $\left.\psi(x, z, u)\right)$, Lemma 24 implies that $\overline{\mathbf{s}}^{(x, z)}$ can be written as a convex combination of $K$ or fewer points in $\mathcal{S}^{(x, z)}$ :

$$
\overline{\mathbf{s}}^{(x, z)}=\sum_{j=1}^{K} \lambda_{j} \mathbf{s}_{j}^{(x, z)},
$$

where $\mathbf{s}_{1}^{(x, z)}, \ldots, \mathbf{s}_{K}^{(x, z)} \in \mathcal{S}^{(x, z)}$ and the coefficients $\lambda_{1}, \ldots \lambda_{K} \in[0,1]$ sum to 1 . Let $u_{1}^{(x, z)}, \ldots, u_{K}^{(x, z)} \in \mathcal{U}$ be preimages of $\mathbf{s}_{1}^{(x, z)}, \ldots, \mathbf{s}_{K}^{(x, z)}$ so

$$
h^{(x, z)}\left(u_{j}^{(x, z)}\right)=\mathbf{s}_{j}^{(x, z)}, \quad j \in\{1, \ldots, K\} .
$$


We can now define the function $\tilde{\psi}$ as mapping every pair $(x, z) \in \mathcal{X} \times \mathcal{Z}$ and every $j \in\{1, \ldots, K\}$ to

$$
\tilde{\psi}(x, z, j) \triangleq \psi\left(x, z, u_{j}^{(x, z)}\right) .
$$

And we define the random variable $\tilde{U}$ to be conditionally independent of $Y$ given $(X, Z)$ with the conditional law

$$
\operatorname{Pr}[\tilde{U}=j \mid X=x, Z=z]=\lambda_{j}^{(x, z)}, \quad j \in\{1, \ldots, K\} .
$$

The Markov condition (289) thus holds by definition. Moreover, (292), (293), and (296)-(300) combine to prove that $\tilde{U}$ and $\tilde{\psi}$ also satisfy the $K$ distortion constraints in (290): denoting the $k$-th component of the vector $\mathbf{s}_{j}$ by $s_{j, k}$, for $j, k \in\{1, \ldots, K\}$,

$$
\begin{aligned}
& \mathrm{E}\left[d_{k}(x, \phi(Y, z), \tilde{\psi}(x, z, \tilde{U}))\right] \\
& =\sum_{j=1}^{K} \lambda_{j} \mathrm{E}\left[d_{k}(x, \phi(Y, z), \tilde{\psi}(x, z, j))\right] \\
& =\sum_{j=1}^{K} \lambda_{j} \mathrm{E}\left[d_{k}\left(x, \phi(Y, z), \psi\left(x, z, u_{j}^{(x, z)}\right)\right)\right] \\
& =\sum_{j=1}^{K} \lambda_{j} s_{j, k}^{(x, z)} \\
& =\bar{s}_{k}^{(x, z)} \\
& \leq D_{k}^{(x, z)},
\end{aligned}
$$

where the first equality holds by (300), the second equality by (299), the third equality by (293) and (298), the fourth equality by (297), and the inequality at the end by (296). Finally, from (305) we conclude that

$$
\begin{aligned}
& \mathrm{E}\left[d_{k}(X, \phi(Y, Z), \tilde{\psi}(X, Z, \tilde{U}))\right] \\
& \quad=\sum_{x \in \mathcal{X}, z \in \mathcal{Z}} \operatorname{Pr}[X=x, Z=z] \mathrm{E}\left[d_{k}(x, \phi(Y, z), \tilde{\psi}(x, z, \tilde{U}))\right] \\
& \quad \leq \sum_{x \in \mathcal{X}, z \in \mathcal{Z}} \operatorname{Pr}[X=x, Z=z] D_{k}^{(x, z)} \\
& \leq D_{k}
\end{aligned}
$$

where the last inequality follows from the definition of $D_{k}^{(x, z)}$ in (292) and the fact that the tuple $(U, Z, \phi, \psi)$ satisfies the original distortion constraints in (160).

\section{ACKNOWLEDGMENT}

We acknowledge helpful discussions with Gerhard Kramer.

\section{REFERENCES}

[1] A. Wyner and J. Ziv, "The rate-distortion function for source coding with side information at the decoder," IEEE Trans. Inf. Theory, vol. 22, no. 1, pp. 1-10, Jan. 1976.

[2] Y. Steinberg, "Coding and common reconstruction," IEEE Trans. Inf. Theory, vol. 55, no. 11, pp. 4995-5010, Nov. 2009.

[3] K. Kittichokechai, T. J. Oechtering, and M. Skoglund, "Source coding with common reconstruction and action-dependent side information," in Proc. IEEE Inf. Theory Workshop, Dublin, Ireland, Aug./Sep. 2010, pp. $1-5$.
[4] R. Timo, A. Grant, and G. Kramer, "Rate distortion functions for source coding with complementary side information," in Proc. IEEE Int. Symp. Inf. Theory, St. Petersburg, Russia, Jul./Aug. 2011, pp. 2934-2938.

[5] R. Timo, A. Grant, and G. Kramer, "Lossy broadcasting with complementary side information," IEEE Trans. Inf. Theory, vol. 59, no. 1, pp. 104-131, Jan. 2013.

[6] B. Ahmadi, R. Tandon, O. Simeone, and H. V. Poor, "Heegard-Berger and cascade source coding problems with common reconstruction constraints," IEEE Trans. Inf. Theory, vol. 53, no. 3, pp. 1458-1474, Mar. 2013.

[7] B. N. Vellambi and R. Timo, "The Heegard-Berger problem with common receiver reconstructions," in Proc. IEEE Inf. Theory Workshop, Seville, Spain, Sep. 2013, pp. 1-5.

[8] F. E. Rezagah and E. Erkip, "Interactive function computation with reconstruction constraints," in Proc. 51st Annu. Allerton Conf. Commun., Control, Comput., Monticello, IL, USA, Oct. 2013, pp. 896-900.

[9] A. El Gamal and Y.-H. Kim, Network Information Theory. Cambridge U.K.: Cambridge Univ. Press, 2011.

[10] F. M. J. Willems, "Computation of the Wyner-Ziv rate-distortion function," Auxilary thesis, Dept. Wiskunde, Katholieke Univ. Leuven, Leuven, Belgium, Oct. 1982.

[11] F. Dupuis, W. Yu, and F. M. J. Willems, "Blahut-Arimoto algorithms for computing channel capacity and rate-distortion with side information," in Proc. IEEE Int. Symp. Inf. Theory, Chicago, IL, USA, Jun./Jul. 2004, p. 181.

[12] W. Gu, "On achievable rate regions for source coding over networks," Ph.D. dissertation, Dept. Math. Sci. California Inst. Technol., Pasadena, CA, USA, Aug. 2009.

[13] S. Jalali and M. Effros, "Separation of source-network coding and channel coding in wireline networks," submitted to IEEE Trans. Inf. Theory. Available at http://arxiv.org/abs/1110.3559.

[14] T. Cover and M. Chiang, "Duality between channel capacity and rate distortion with two-sided state information," IEEE Trans. Inf. Theory, vol. 48, no. 6, pp. 1629-1638, Jun. 2002.

[15] A. Kaspi, "Two-way source coding with a fidelity criterion," IEEE Trans. Inf. Theory, vol. 31, no. 6, pp. 735-740, Nov. 1985.

[16] J. Thomas, "Feedback can at most double Gaussian multiple access channel capacity (Corresp.)," IEEE Trans. Inf. Theory, vol. 33, no. 5, pp. 711-716, Sep. 1987.

[17] R. T. Rockafellar, Convex Analysis. Princeton, NJ, USA: Princeton Univ. Press, 1970.

[18] R. Gray, "A new class of lower bounds to information rates of stationary sources via conditional rate-distortion functions," IEEE Trans. Inf. Theory, vol. 19, no. 4, pp. 480-489, Jul. 1973.

[19] C. E. Shannon, "Probability of error for optimal codes in a Gaussian channel," Bell Syst. Tech. J., vol. 38, no. 3, pp. 611-656, 1959.

[20] A. Lapidoth and S. Tinguely, "Sending a bivariate Gaussian over a Gaussian MAC," IEEE Trans. Inf. Theory, vol. 56, no. 6 , pp. 2714-2752, Jun. 2010.

Amos Lapidoth (S'89-M'95-SM'00-F'04) received the B.A. degree in mathematics (summa cum laude, 1986), the B.Sc. degree in electrical engineering (summa cum laude, 1986), and the M.Sc. degree in electrical engineering (1990) all from the Technion-Israel Institute of Technology. He received the Ph.D. degree in electrical engineering from Stanford University in 1995.

In the years 1995-1999 he was an Assistant and Associate Professor at the Department of Electrical Engineering and Computer Science at the Massachusetts Institute of Technology, and was the KDD Career Development Associate Professor in Communications and Technology. He is now Professor of Information Theory at ETH Zurich in Switzerland. He is the author of the book A Foundation in Digital Communication published by Cambridge University Press in 2009. His research interests are in digital communications and information theory.

Dr. Lapidoth served in the years 2003-2004 and 2009 as Associate Editor for Shannon Theory for the IEEE TRANSACTIONS ON INFORMATION THEORY. 
Andreas Malär received the M.Sc. degree in electrical engineering from ETH Zurich in early 2011. In 2011 he was a research assistant at the Signal and Information Processing Laboratory (ISI) at ETH Zurich. Since 2006 he is CEO of Malcom AG, Zurich, Switzerland. His current research interests are in front-end engineering and responsive design.
Michèle Wigger (S'05-M'09) received the M.Sc. degree in electrical engineering (with distinction) and the Ph.D. degree in electrical engineering both from ETH Zurich in 2003 and 2008, respectively. In 2009 she was a postdoctoral researcher at the ITA center at the University of California, San Diego. Since December 2009 she is an Assistant Professor at Telecom ParisTech, in Paris, France. Her research interests are in information and communications theory. 